\title{
Seismology on Mars
}

\author{
Don L. Anderson, ${ }^{1}$ W. F. Miller,${ }^{1}$ G. V. Latham, ${ }^{2}$ Y. Nakamura, ${ }^{2}$ M. N. Toksöz,${ }^{3}$ A. M. Dainty, ${ }^{3}$ \\ F. K. Duennebier, ${ }^{4}$ A. R. Lazarewicz, ${ }^{4}$ R. L. Kovach, ${ }^{5}$ and T. C. D. Knight ${ }^{6}$
}

\begin{abstract}
A three-axis short-period seismometer has been operating on the surface of Mars in the Utopia Planitia region since September 4, 1976. During the first 5 months of operation, approximately 640 hours of highquality data, uncontaminated by lander or wind noise, have been obtained. The detection threshold is estimated to be magnitude 3 to about $200 \mathrm{~km}$ and about 6.5 for the planet as a whole. No large events have been seen during this period, a result indicating that Mars is less seismically active than earth. Wind is the major source of noise during the day, although the noise level was at or below the sensitivity threshold of the seismometer for most of the night during the early part of the mission. Winds and therefore the seismic background started to intrude into the nighttime hours starting on sol 119 (a sol is a Martian day). The seismic background correlates well with wind velocity and is proportional to the square of the wind velocity, as is appropriate for turbulent flow. The seismic envelope power spectral density is proportional to frequency to the -0.66 to -0.90 power during windy periods. A possible local seismic event was detected on sol 80 . No wind data were obtained at the time, so a wind disturbance cannot be ruled out. However, this event has some unusual characteristics and is similar to local events recorded on earth through a Viking seismometer system. If it is interpreted as a natural seismic event, it has a magnitude of 3 and a distance of $110 \mathrm{~km}$. Preliminary interpretation of later arrivals in the signal suggest a crustal thickness of $15 \mathrm{~km}$ at the Utopia Planitia site which is within the range of crustal models derived from the gravity field. More events must be recorded before a firm interpretation can be made of seismicity or crustal structure. One firm conclusion is that the natural background noise on Mars is low and that the wind is the prime noise source. It will be possible to reduce this noise by a factor of $10^{3}$ on future missions by removing the seismometer from the lander, operation of an extremely sensitive seismometer thus being possible on the surface.
\end{abstract}

\section{INTRODUCTION}

Because the primary emphasis on landed Viking science was on biology, organic chemistry, imagery, and meteorology, other areas such as surface chemistry, petrology, and geophysics were mostly relegated to future missions. The exceptions were the inorganic analysis experiment, the magnet experiment, and the seismometer. However, these studies were limited to reconnaissance measurements.

The ultimate goals of a seismic experiment are to determine the dynamics and internal structure of a planet. These are relevant to the composition and evolution of the interior. Before a comprehensive seismic experiment can be considered, however, it is necessary to establish the background noise level of the planet, the level, nature, and location of the seismicity, and the nature of the seismic signals. Estimates of some of these parameters have been obtained by the Viking seismometer. The ultimate goal, however, can only be accomplished with a network of sensitive seismometers deployed so as to minimize artificial sources of noise and wind-induced vibrations.

The Viking seismic experiment design was constrained by strict weight, power, and data allocations and perturbed by the conflicting demands of the other on-board experiments. The weight constraint precluded an ultrasensitive seismometer of the Apollo class or a broadband seismometer. The original desire to offload the seismometer was sacrificed because of the

${ }^{1}$ Division of Geological and Planetary Sciences, California Institute of Technology, Pasadena, California 91125.

${ }^{2}$ University of Texas, Galveston, Texas 77550 .

${ }^{3}$ Massachusetts Institute of Technology, Cambridge, Massachusetts 02139 .

${ }^{4}$ University of Hawaii, Honolulu, Hawaii 96822.

${ }^{5}$ Stanford University, Palo Alto, California 94305.

6 Martin Marietta Aerospace Corp., Denver, Colorado 80201.

Copyright (C) 1977 by the American Geophysical Union.

Paper number 7 S0408. weight and complexity penalty of such an operation; thus an on-board location was dictated that immediately increased the noise level because of lander and wind activity (by at least 3 orders of magnitude). The data constraint required severe data compression using an on-board data processing capability and thus also a weight and power penalty. The most severe constraints on the Viking seismic experiment were limited data allocations and the on-board location of the seismometer.

These various constraints and trade-offs led to the design of a short-period three-component seismometer with on-board data compaction and triggering to optimize the data return [Anderson et al., 1972]. The objectives were (1) to characterize the seismic noise environment at the landing sites, (2) to detect local events in the vicinity of the lander, and (3) to detect large events at teleseismic distances.

Under optimal conditions it would also be possible to determine the following: (1) the approximate distance of events by the separation of the various seismic phases, (2) the direction of events with a $180^{\circ}$ ambiguity, (3) the attenuation and scattering properties of the crust to determine if the crust is lunarlike or earthlike in these characteristics (which are related to the volatile content), and (4) an estimate of crustal thickness if crustal and reflected phases can be identified. The Viking 1 seismometer failed to uncage, and no useful data were returned. The Viking 2 seismometer, emplaced on the surface of Mars in the Utopia Planitia region, $47.9^{\circ} \mathrm{N}, 225.9^{\circ} \mathrm{W}$, successfully uncaged and has been operating nominally since that time [Anderson et al., 1976].

\section{State of Stress in the Martian Interior}

The regional topography of the earth is in approximate isostatic balance, and regions of partial compensation are generally the most seismically active. Except for the nearside mascons the moon is also in equilibrium. Both bodies are seismically active, although the moon is many orders of magnitude less active than the earth. Large areas of Mars seem to 
be only partially compensated [Phillips and Saunders, 1975] and could be seismically active. These are the younger areas which include the Tharsis plateau and the adjacent low areas of Chryse and Amazonis. Stresses implied by lack of compensation and dynamic stresses in the earth and the moon are in the 10- to 100-bar range. Stress drops in earthquakes are also in this range. Laboratory measurements on crustal rocks at modest pressure give short-term strengths of kilobars, but rocks creep at high temperatures at relatively low stress levels. Stresses in the crust of a planet therefore tend to decrease with time unless they are rejuvenated by plate tectonic motions, convective drag, or thermoelastic stresses caused by heating or cooling. Phillips and Tiernan [1977] argue that the Tharsis gravity anomaly represents a long-wavelength stress supported by the lithosphere or the asthenosphere for $10^{8}-10^{9}$ years, implying stresses in the mantle of 100 bars or stresses in the lithosphere of a few kilobars. They favor at least partial dynamic support of Tharsis by mechanisms such as mantle convection or viscous and thermoelastic stresses associated with magmatic activity. The existence of large stresses in the Tharsis region, at least at the time of formation, is also implied by the pattern of lineaments, including apparent grabens and the great canyon, aligned generally radially with respect to the plateau summit [Blasius and Cutts, 1976]. The depth of compensation of the Martian topography is of the order of $150 \mathrm{~km}$, and about $3 \mathrm{~km}$ of Tharsis is presently uncompensated [Phillips and Saunders, 1975]. It is likely that the uncompensated areas of Mars are seismically active, since the stresses are similar or greater than stresses in the earth and moon. This is true whether the planet is striving toward a state of isostatic equilibrium or whether the stresses are being rejuvenated. Most of the seismic activity on earth is associated with plate margins, although moderate to large (but infrequent) earthquakes also occur in plate interiors of both oceanic and continental plates. Plate motions on the moon and Mars are certainly less pronounced than on earth, so we might also expect Mars to be less seismically active than earth. On the other hand, the larger stresses required to support the topography and the gravity field imply the potential for seismic activity even if creep and flow occur. In the earth and in the laboratory, deformation proceeds by both slow aseismic processes (creep) and rapid seismic processes (earthquakes). The latter dominate at low temperatures such as exist in the lithosphere.

The variations of gravity and topography suggest that the seismicity of Mars is not uniform and that it is likely to be highest near Tharsis and its environs and similar young uncompensated constructs. The Viking 2 lander is approximately $110^{\circ}$ from Chryse Planitia (VL-1 site) and $25^{\circ}$ from Elysium Mons. Marsquakes at these locations would have to be quite large to be detected by the Viking 2 seismometer. In summary, we expect Mars to be a tectonically active planet, but the seismicity may be localized.

\section{MODELS FOR THE INTERIOR OF MARS}

Interpretation of Martian seismic data requires an a priori model of the planet's interior. Such a model must be calculated on the basis of available direct data, cosmochemical considerations, equations of state, and theoretical modeling of the planetary interior and its thermal evolution. Such efforts have been made by a number of investigators [Urey, 1952; Anderson, 1972; Anderson and Kovach, 1967; Kovach and Anderson, 1965; Binder, 1969; Binder and Davis, 1973; Johnston et al., 1974; Johnston and Toksöz, 1977; Ringwood and Clark, 1971; Toksöz and Johnston, 1977]. Here we will briefly review the results most relevant to the seismic experiment. The only direct evidence concerning the internal structure of Mars prior to the Viking landings was the mean density, moment of inertia, topography, and gravity field. It is possible to calculate models of the Martian interior with plausible chemical models and temperature profiles that satisfy these few constraints. However, the process is highly nonunique.

The mean density of Mars, corrected for pressure, is less than that of earth, Venus, and Mercury but greater than that of the moon. This implies that Mars has a smaller total Fe-Ni content than do the other terrestrial planets and more than does the moon. Plausible models for Mars can be constructed which have solar or chondritic values for Fe [Anderson, 1972]. Mars is the only terrestrial planet, including the moon, which could have chondritic abundances of iron, and it is therefore likely to contain a larger proportion of volatiles such as water-, sulfur-, and potassium-bearing phases. The densities and compositions of the planets are consistent with the view that the volatile content increases with distance from the sun [Anderson, 1971]. This can be understood in terms of a primitive solar nebula in which the decreasing temperature away from the sun controlled the composition of the condensates which were available for incorporation into the various planets. In this view, Mars should be more volatile rich than the other terrestrial planets. Volatiles in this context include FeS, hydrous minerals, water, $\mathrm{K}_{2} \mathrm{O}$, and $\mathrm{Na}_{2} \mathrm{O}$. Low condensation temperatures also imply a higher oxidation state for the available iron; i.e., more $\mathrm{Fe}$ should be available as $\mathrm{FeS}, \mathrm{FeO}, \mathrm{Fe}_{2} \mathrm{O}_{9}$, and $\mathrm{Fe}_{3} \mathrm{O}_{4}$ than is the case for the primitive earth. The core of Mars therefore is probably richer in FeS, and the mantle is richer in $\mathrm{FeO}$ than is the case for the earth, where more free $\mathrm{Fe}$ is available. A large $\mathrm{Fe}-\mathrm{FeS}$ core, an $\mathrm{FeO}$-rich mantle, and a thick crust $\left(\mathrm{Na}_{2} \mathrm{O}, \mathrm{K}_{2} \mathrm{O}\right.$, and hydrous minerals $)$ are therefore expected. On the other hand, the relative proportions of $\mathrm{CaO}$ and $\mathrm{Al}_{2} \mathrm{O}_{3}$, components of crustal minerals, are expected to be less, and the total crustal thickness may be buffered by the availability of these constituents. However, $\mathrm{SiO}_{2}$ and lowmelting-point $\mathrm{FeO}$-bearing compounds probably dominate the crustal composition.

With such broad chemical constraints, mean density $(\bar{\rho}=$ $\left.3.96 \mathrm{~g} / \mathrm{cm}^{3}\right)$, and moment of inertia factor $\left(C / M R^{2}=0.365\right.$, Reasenberg [1977]) and under the assumption of a differentiated planet it is possible to trade off the size and density of the core and density of the mantle [Anderson, 1972; Binder and Davis, 1973; Johnston et al., 1974; Johnston and Toksöz, 1977]. Most of these models favor an $\mathrm{FeO}$ enrichment of the Martian mantle relative to the mantle of the earth. Anderson [1972] concluded that Mars has a total iron content of about $25 \mathrm{wt} \%$, which is significantly less than the iron content of earth, Mercury, or Venus but is close to the total iron content of ordinary and carbonaceous chondrites. The high zero-pressure density of the mantle suggests a relatively high $\mathrm{FeO}$ content in the silicates of the Martian mantle. The radius of the core can range from as small as one-third the radius of the planet for an iron core, or a core similar in composition to the earth's core, to more than half the radius of the planet if it is pure FeS. With chondritic abundances of Fe-FeS the size of the core would be about $45 \%$ of the planet's radius, or about $12 \%$ by mass. A small dense core would imply a high-temperature origin or early history because of the high melting temperature of Fe$\mathrm{Ni}$, while a larger light core, presumably rich in sulfur, would allow a cooler early history, since sulfur substantially reduces the melting temperature. A satisfactory model for the interior of Mars can be obtained by exposing ordinary chondrites to 
modest temperatures, melting the $\mathrm{Fe}-\mathrm{FeS}$, and removing most of it to the core [Anderson, 1972]. This would make the Martian core substantially more $\mathbf{S}$ rich than the terrestrial core and would favor the larger core radius. Alternatively, Mars could be a mixture of carbonaceous and ordinary chondrites (or hypersthene or bronzite chondrites) and satisfy the mean density and moment of inertia with partial removal of Fe-Ni-S from the silicate phase to the core.

The trade-offs between core size, composition, and mantle density are given by Anderson [1972] and Johnston and Toksöz [1977]. Under the assumption of an uncertainty of \pm 0.005 in the moment of inertia factor the core radius in these models could vary between about 1300 and $1800 \mathrm{~km}$, and the mantle density, corrected to $0^{\circ} \mathrm{C}$ and 1-bar pressure, between $\rho_{m}$ of 3.4 and $3.6 \mathrm{~g} / \mathrm{cm}^{3}$. It is obvious that a direct determination of the size of the core by seismic means could constrain the overall composition of the planet.

The topography and gravity fields of Mars indicate that parts of Mars are grossly out of hydrostatic equilibrium and that the crust is highly variable in thickness. If variations in the gravity field are attributed to variations in crustal thickness, with a constant density ratio between crust and mantle, then reasonable values of the density contrast $\left(<0.8 \mathrm{~g} / \mathrm{cm}^{3}\right)$ imply that the average crustal thickness is at least $30 \mathrm{~km}$ (W. M. Kaula, personal communication, 1976). This minimal bound is based on the assumption of zero crustal thickness in the Hellas basin (R. Phillips, personal communication, 1976). An impact large enough to excavate the Hellas basin would easily remove a $30-\mathrm{km}$-thick crustal layer (T. J. Ahrens, personal communication, 1976). This minimal average crustal thickness on Mars gives a crust/planet mass ratio that is more than 5 times the terrestrial value, indicating a well-differentiated planet.

The crust of the earth is enriched in $\mathrm{CaO}, \mathrm{Al}_{2} \mathrm{O}_{3}, \mathrm{~K}_{2} \mathrm{O}$, and $\mathrm{Na}_{2} \mathrm{O}$ in comparison to the mantle. Ionic radii considerations and experimental petrological results suggest that the crust of any planet will be enriched in these constituents. A minimal average crustal thickness for a fully differentiated chondritic planet can be obtained by removing all of the $\mathrm{CaO}$ possible, with the available $\mathrm{Al}_{2} \mathrm{O}_{3}$, as anorthite to the surface. This gives a crustal thickness of about $100 \mathrm{~km}$ for Mars. Incomplete differentiation and retention of $\mathrm{CaO}$ and $\mathrm{Al}_{2} \mathrm{O}_{3}$ in the mantle will reduce this value, which is likely to be the absolute upper bound.

The average thickness of the crust of the earth is $15 \mathrm{~km}$, which amounts to $0.4 \%$ of the mass of the earth. The crustal thickness is $5-10 \mathrm{~km}$ under oceans and $30-50 \mathrm{~km}$ under older continental shields. The thickness of the crust increases with age. The volume of the crust increases only slightly if presently active subducted regions are taken into account. The situation on the earth is complicated, since new crust is constantly being created at the midoceanic ridges and consumed at island arcs. It is probable that some of the crust is being recycled. If the present rate of crustal genesis was constant over the age of the earth and none of the crust was recycled, then $17 \%$ of the earth would be crustal material.

The moon apparently has a mean crustal thickness greater than that of the earth. If the average composition of the moon is similar to chondrites minus the Fe-Ni-S, then the crust could be as thick as an average of $62 \mathrm{~km}$. This is about the thickness of the crust determined by seismic experiments on the frontside of the moon [Toksöz et al., 1974] but less than the average thickness inferred from the gravity field [Bills and Ferrari, 1977a]. An alternative model based on a Ca-Al-rich moon

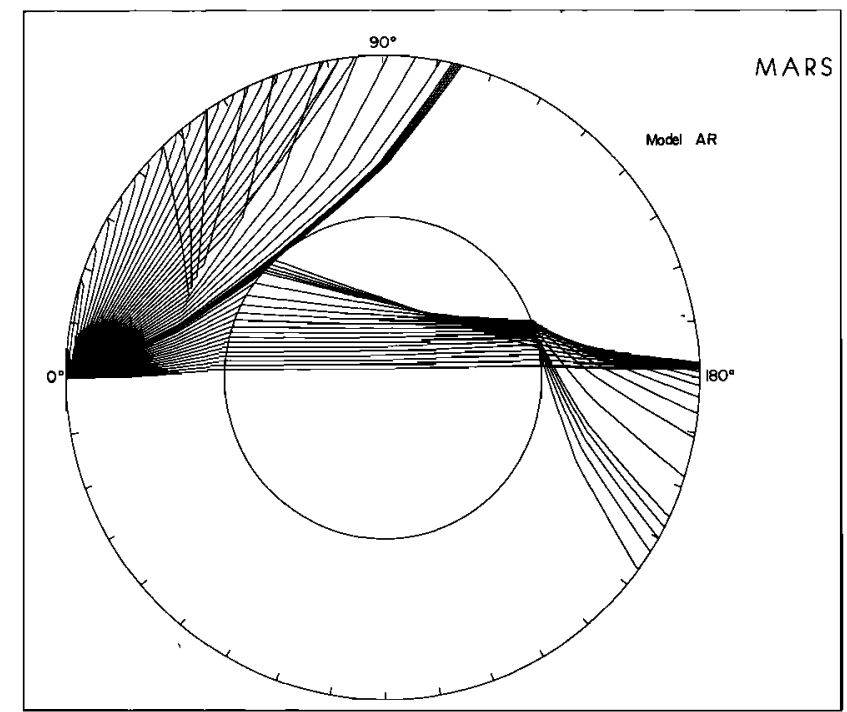

Fig. 1. Seismic ray paths for compressional waves through the interior of a theoretical Martian model that satisfies the mean density and moment of inertia. The 'mantle' is homogeneous in composition, the olivine-spinel transition causing a discontinuity at a $1100-\mathrm{km}$ depth. Note the shadow zone at the surface caused by the decrease in velocity in the core. The core is assumed to be molten. Parameters for the model are shown in Figure 2.

[Anderson, 1973] can yield a much greater crustal thickness.

The surface reflectance spectra of Phobos and Deimos and the low density of Phobos are also consistent with the fact that these bodies are similar to carbonaceous chondrites. Even if these are captured bodies, the suggestion is that Mars may have accreted from relatively volatile rich material. The state of water on Mars is unknown, but the evidence for running water at the surface early in its history and the inability of substantial amounts of water to escape from the atmosphere indicate that there should be free or bound water in the interior.

Atmospheric analyses suggest that Mars is a less outgassed planet than earth [Owen and Biemann, 1976], but the high ratio of ${ }^{40} \mathrm{Ar}$ relative to the other inert gases suggests that the Martian crust is enriched in ${ }^{40} \mathrm{~K}$ in relation to the earth's crust.

The volatile content of a planet's interior along with the temperature of the interior are the two most important factors that control the attenuation of seismic waves. The attenuation properties of the Martian mantle may be similar to those of the earth's mantle with the possible exception of the low $Q$ (high attenuation) zones of the earth's asthenosphere. Thus we would expect seismic waves to propagate through the Martian interior with similar or greater efficiency than they do on earth, particularly if the lithosphere is as thick as is implied by isostatic calculations [Phillips and Saunders, 1975]. Compressional wave ray paths are shown in Figure 1 for a typical Martian model based on an FeS core composition. Note the large shadow zone due to the low-velocity core. It is important to point out that these models are nonunique and actual seismic data are required to determine the internal structure and composition of Mars. The velocities and density models used to calculate the ray paths in Figure 1 are shown in Figure 2.

\section{Crustal Structure of Mars}

It is clear from the topography and gravity field of Mars that the crustal layer is highly nonuniform. Attempts have been 


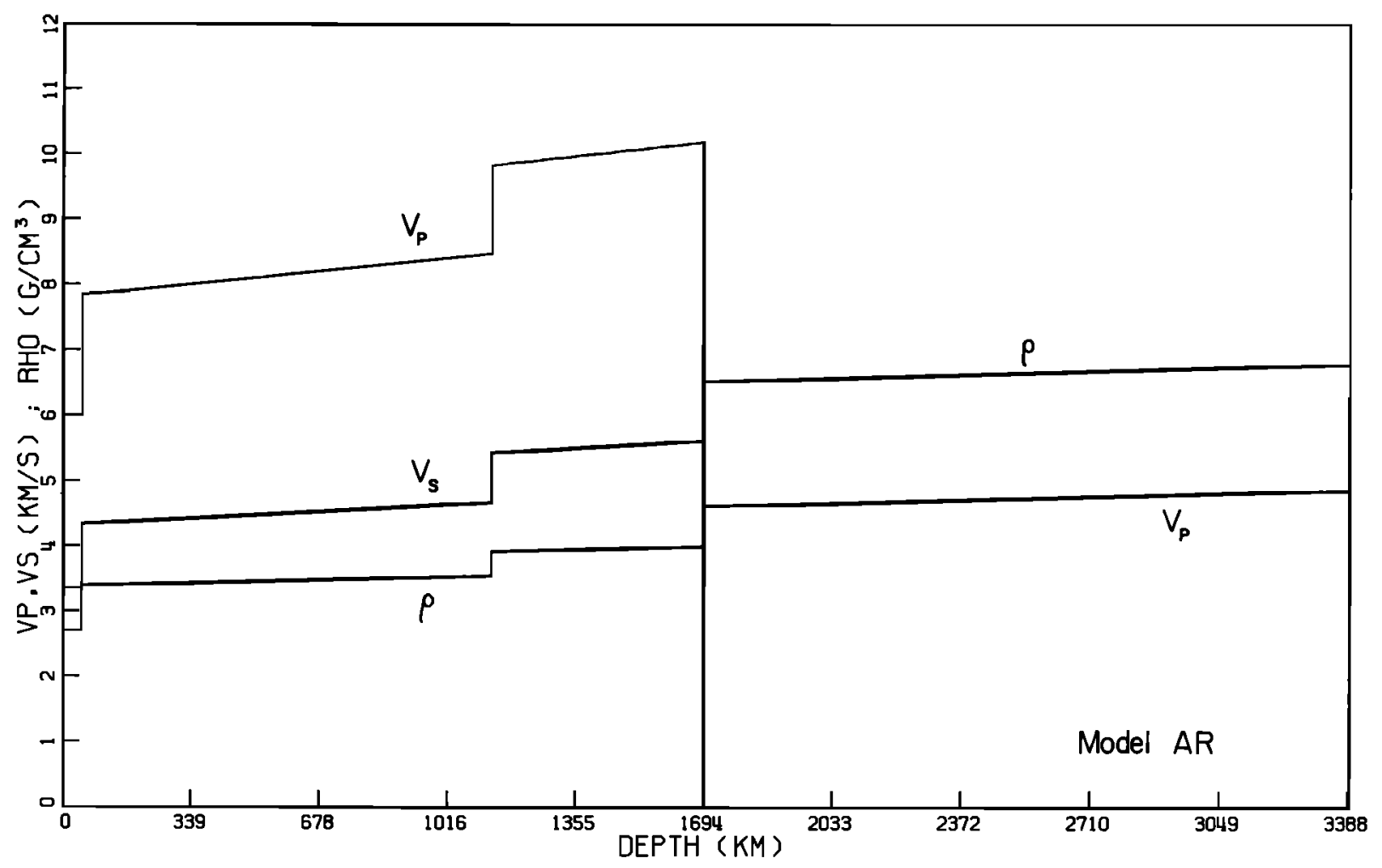

Fig. 2. Theoretical velocities and density in the interior of Mars. This model satisfies the known constraints of mean density and moment of inertia. The core size can be increased or decreased if the density of the core relative to that of the mantle is decreased or increased, respectively. Data used in this model are from Anderson [1.972], Okal and Anderson [1977], and Johnston et al. [1974].

made to infer variations in crustal thickness [Phillips et al., 1973; Phillips and Tiernan, 1977; Bills and Ferrari, 1977b], but the process is nonunique unless it can be tied to a direct measurement of the crustal thickness made by seismic methods. Interpretation of the Bouguer gravity field involves both the crustal thickness and the crust/mantle density ratio. Usually, the mean crustal thickness and the density contrast are assumed, and the Bouguer anomalies are interpreted in terms of deviations of the crustal thickness from the mean. Figure 3 gives a crustal thickness (isopach) map of Mars for an assumed mean crustal thickness of $40 \mathrm{~km}$ and a density contrast of $0.6 \mathrm{~g} / \mathrm{cm}^{3}$ [Bills and Ferrari, 1977a]. Note that the thickest crust, $75 \mathrm{~km}$, is under Tharsis at the head of Valles Marineris and the thinnest crust, $10 \mathrm{~km}$, is under Hellas. A second crustal map for an assumed mean crustal thickness of $100 \mathrm{~km}$ is shown in Figure A-1 of the microfiche appendix. ${ }^{1}$

One way to bound the problem is to assume zero crustal thick ness under the Hellas basin. If this large circular feature is of impact origin, deep excavation can be expected which would remove most, if not all, of the crustal material. For a density contrast of $0.3 \mathrm{~g} / \mathrm{cm}^{3}$ the mean crustal thickness is about $65 \mathrm{~km}$, and the thickness at the Viking 2 site would be $10-20 \mathrm{~km}$. A higher density contrast would decrease the mean thickness and decrease the variation in crustal thickness. The maximum crustal thickness is $140 \mathrm{~km}$ for $\Delta \rho=0.3 \mathrm{~g} / \mathrm{cm}^{3}$ and $90 \mathrm{~km}$ for $\Delta \rho=0.6 \mathrm{~g} / \mathrm{cm}^{3}$. These calculations are due to $\mathrm{R}$. Phillips (personal communication, 1976). He has demonstrated that the crust in the vicinity of the Viking 2 landing site

\footnotetext{
${ }^{1}$ Supplement is available with entire article on microfiche. Order from American Geophysical Union, 1909 K Street, N. W., Washington, D. C. Document J77-004; \$1.00. Payment must accompany order.
}

is quite thin in comparison to the rest of the planet. If the crustal thickness could be determined at a single site and if the density contrast is constant, then we could immediately determine the average crustal thickness of the planet and, for example, the crustal thickness under the Tharsis plateau.

An average crustal thickness of even $30 \mathrm{~km}$ would indicate that Mars is an extremely well differentiated planet having a crust/planet mass ratio of over 5 times that for earth. The average thickness would be less if the density contrast were greater. There is evidence that the Martian mantle is denser than the terrestrial mantle [Anderson, 1972], but there is no direct information on the density of the crust. If ice, water, and hydrated minerals are abundant, then the crustal density may be quite low. On the other hand, a hydrous mantle could result in a well-differentiated planet because of the effect of water on melting temperatures and viscosity.

\section{Description of the Viking Seismometer}

The Viking seismometer includes sensors, amplifiers, filters, and electronics for automatic event detection, data compaction, and temporary data storage. The instrument package, shown in Figure 4, is $12 \times 15 \times 12 \mathrm{~cm}$ and weighs $2.2 \mathrm{~kg}$. It is located on top of the lander's equipment bay near the attachment of leg 1 (Figure 5). The nominal power consumption is $3.5 \mathrm{~W}$. The useful frequency range is $0.1-10 \mathrm{~Hz}$ with a minimum ground amplitude resolution of $2 \mathrm{~nm}$ at $3 \mathrm{~Hz}$ and $10 \mathrm{~nm}$ at $1 \mathrm{~Hz}$.

Figure 6 compares the Viking seismometer displacement response in each of its operating modes with the displacement response of a typical station in the U.S. Geological Survey World-Wide Standard Seismograph Network (WWSSN). The 


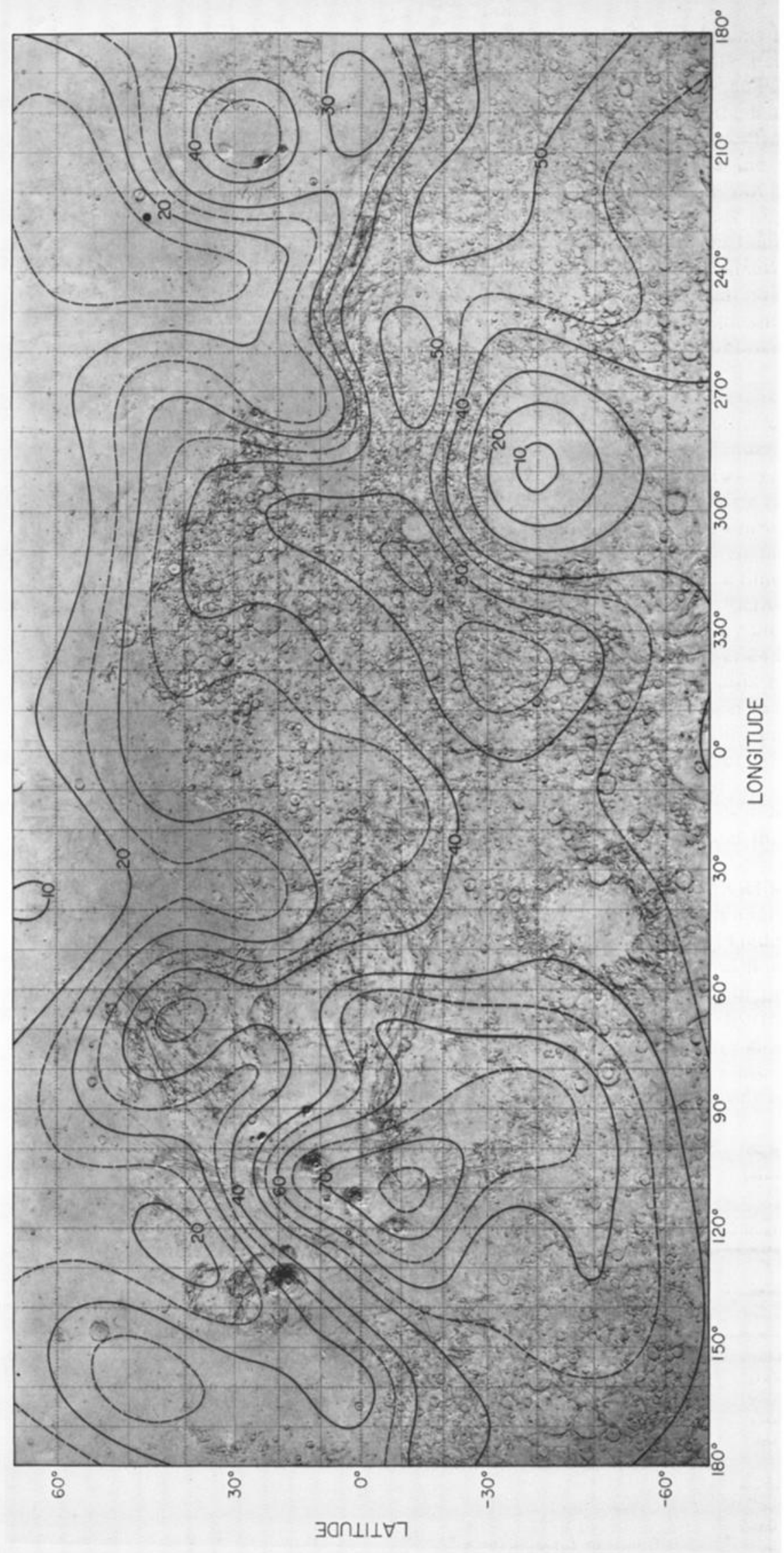

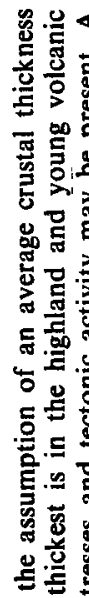

离里送

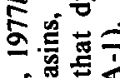

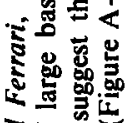

ปี

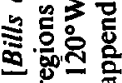

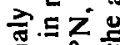

象资

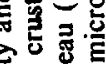

它

要,

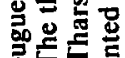

由.

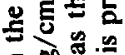

ㄷํㅇํㄷㄹ

造论

ง

西

部总

है

果语

总

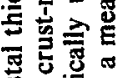

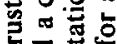

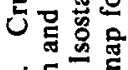

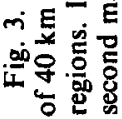




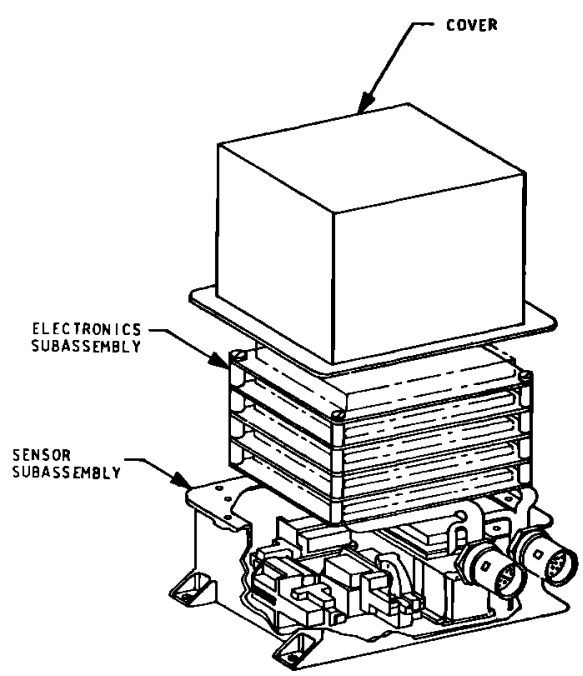

SEISMOMETER ASSEMBLY

Fig. 4. Seismometer package. The dimensions of the unit, sncluding sensors and electronics, are $12 \times 15 \times 12 \mathrm{~cm}$ with a weight of 2.2 $\mathrm{kg}$. The nominal power consumption is $3.5 \mathrm{~W}$.

peak magnification is 218,000 at $3 \mathrm{~Hz}$ when the returned data are plotted at a scale of $0.44 \mathrm{~mm}$ per digital unit of seismometer output. Figure 7 compares the acceleration sensitivity of the Viking seismometer with that of conventional accelerometers, the U.S. Geological Survey WWSSN, and the Apollo lunar instruments. The Viking seismometer has a maximum sensitivity equivalent to that obtainable at a relatively quiet site on earth.
The seismic sensors are three matched, orthogonally mounted (one vertical and two horizontal) inertial seismometers fitted with velocity transducers. One sensor is shown schematically in Figure 8. Each sensor occupies a volume measuring $7.5 \times 3.8 \times 3.8 \mathrm{~cm}$. A 16-g mass-coil assembly is supported on twin booms by two Bendix Free-Flex elastic hinges such that the flat transducer coil is poised between the facing poles of two channel magnets arranged in series. Motion of the frame causes the transducer coil to move in the field of the magnets and generates a signal which is in proportion to the relative velocity of the coil with respect to that of the magnet. The undamped natural frequency of each instrument is $4 \mathrm{~Hz}$, the coefficient of damping is 0.6 , and the generator constant is $177 \mathrm{~V} /(\mathrm{m} / \mathrm{s})$. The natural undamped frequency of the sensors was chosen such that the instrument would meet the constraints of weight and volume and to insure that the sensors would operate over the largest expected tilt of the lander $\left(15^{\circ}\right)$ without the use of any mechanical zeroing adjustments. With this design the horizontal units will tolerate up to $23^{\circ}$ of tilt, and the vertical up to $35^{\circ}$.

The inertial mass of each sensor is individually caged to protect it from the shock and vibration encountered during launch, separation of the lander from the orbiter (the greatest shock peaking at $1200 \mathrm{~g}$ ), and the landing. The caging is provided by spring-loaded plungers which hold the mass firmly against a stop seat. The plungers are secured by a palladiumaluminum fuse wire. Uncaging is accomplished by causing the fuse wire to fail nonexplosively when it is electrically heated. All three axes failed to uncage on the Viking 1 lander. Because the uncaging system was triply redundant except at the interface with the lander and because the seismometer functioned properly in all other aspects, it is believed that the failure was

\section{VIKING LANDED SCIENCE CONFIGURATION}

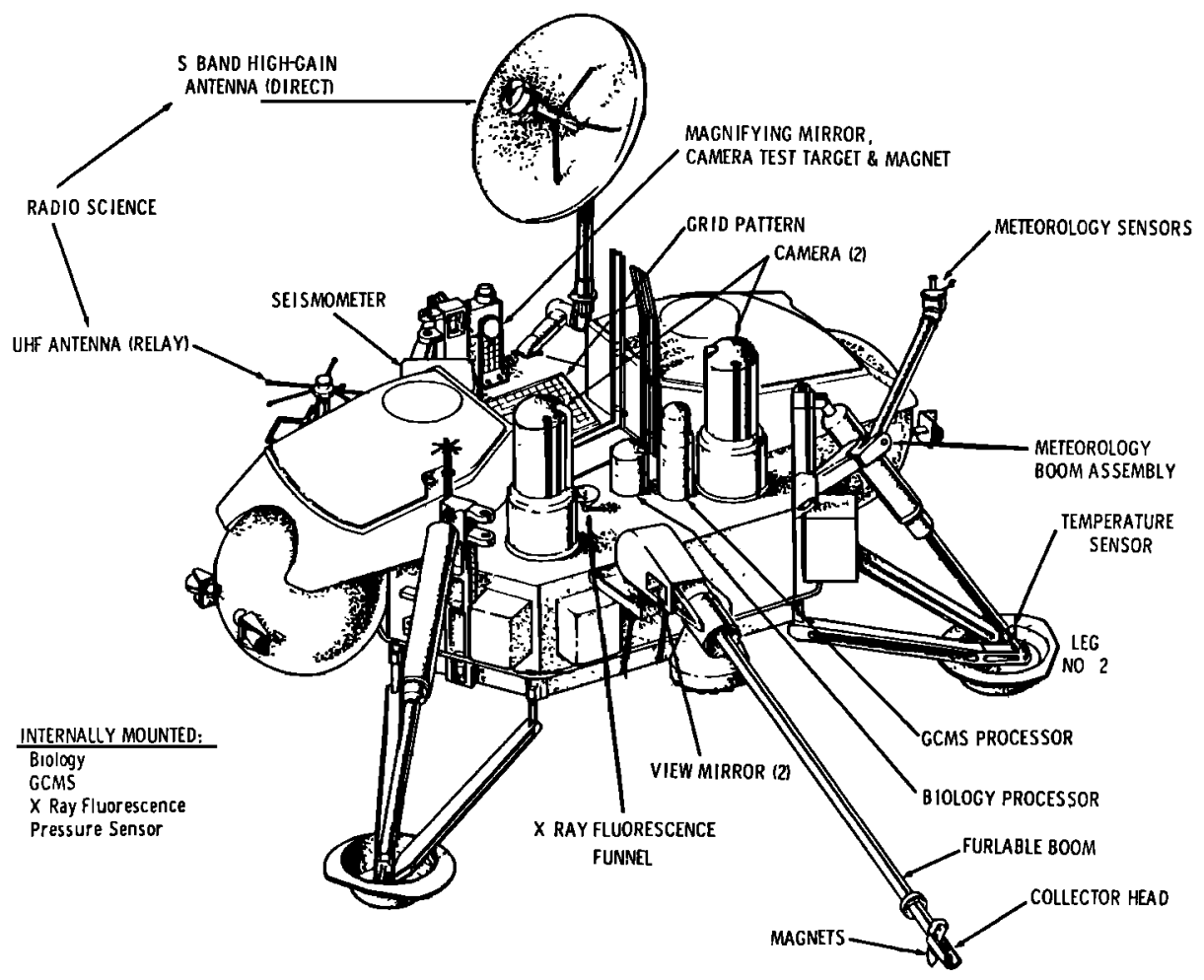

Fig. 5. The Viking lander showing the location of the seismic package and other instruments. The distance from footpad to footpad is about $2.5 \mathrm{~m}$, and the lander mass is $605 \mathrm{~kg}$. 


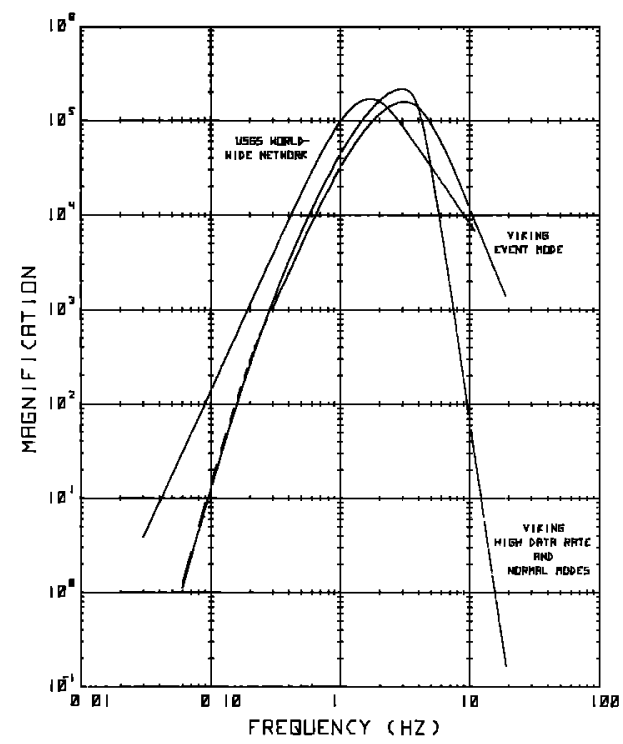

SEISMLMETER MAGNIFICATION

Fig. 6. Magnification of the Viking seismograph in each of the operating modes. The magnifications are based on the assumption that the digitized data are plotted at scales of $0.44,0.51$, and $0.76 \mathrm{~mm} / \mathrm{du}$ for the high data rate, event, and normal modes, respectively. A typical U.S. Geological Survey short-period instrument used in the WWSSN is shown for comparison. at the interface. The Viking 2 lander seismometer uncaged in a nominal fashion.

Each inertial sensor is equipped with a calibration mechanism by which the mass may be magnetically deflected approximately $4 \mu \mathrm{m}$. The deflection and release of the mass in each direction produce a pair of pulse doublets, shown in Figure 9, from which the operation and characteristics of the instrument may be ascertained and which may be used to determine the attitude of the seismometer package relative to the local vertical by measuring the asymmetries of the calibration doublet which are a function of the tilt of the instrument. This comparison of the Viking 2 seismometer calibration doublet with prelaunch calibration data showed the instrument attitude to be $9.5^{\circ} \pm 2.8^{\circ}$ down at an azimuth of $278^{\circ} \pm 17^{\circ}$ from north. This is in agreement with the lander's inertial reference system, which determined the tilt of the lander to be $8.2^{\circ}$ down at an azimuth of $278^{\circ}$ from north.

A block diagram of the instrument is shown in Figure 10. Each axis has an amplifier with a bandwidth of $0.2-1.2 \mathrm{~Hz}$ and an amplification, which is selectable upon earth command, from $6 \times 10^{3}$ to $4 \times 10^{5}$ in six increments. After amplification, prefiltering, and analog multiplexing, the seismic signals are converted to a 7-bit plus sign digital word at the rate of 121.21 samples per second by a dual-slope integrating analog-todigital converter. The subsequent digital processing of the data includes filtering, averaging, compression, event detection, and buffer memory storage. These functions, as well as digital multiplexing, command decoding, timing, and control, are implemented in custom large-scale-integrated (LSI) circuitry.

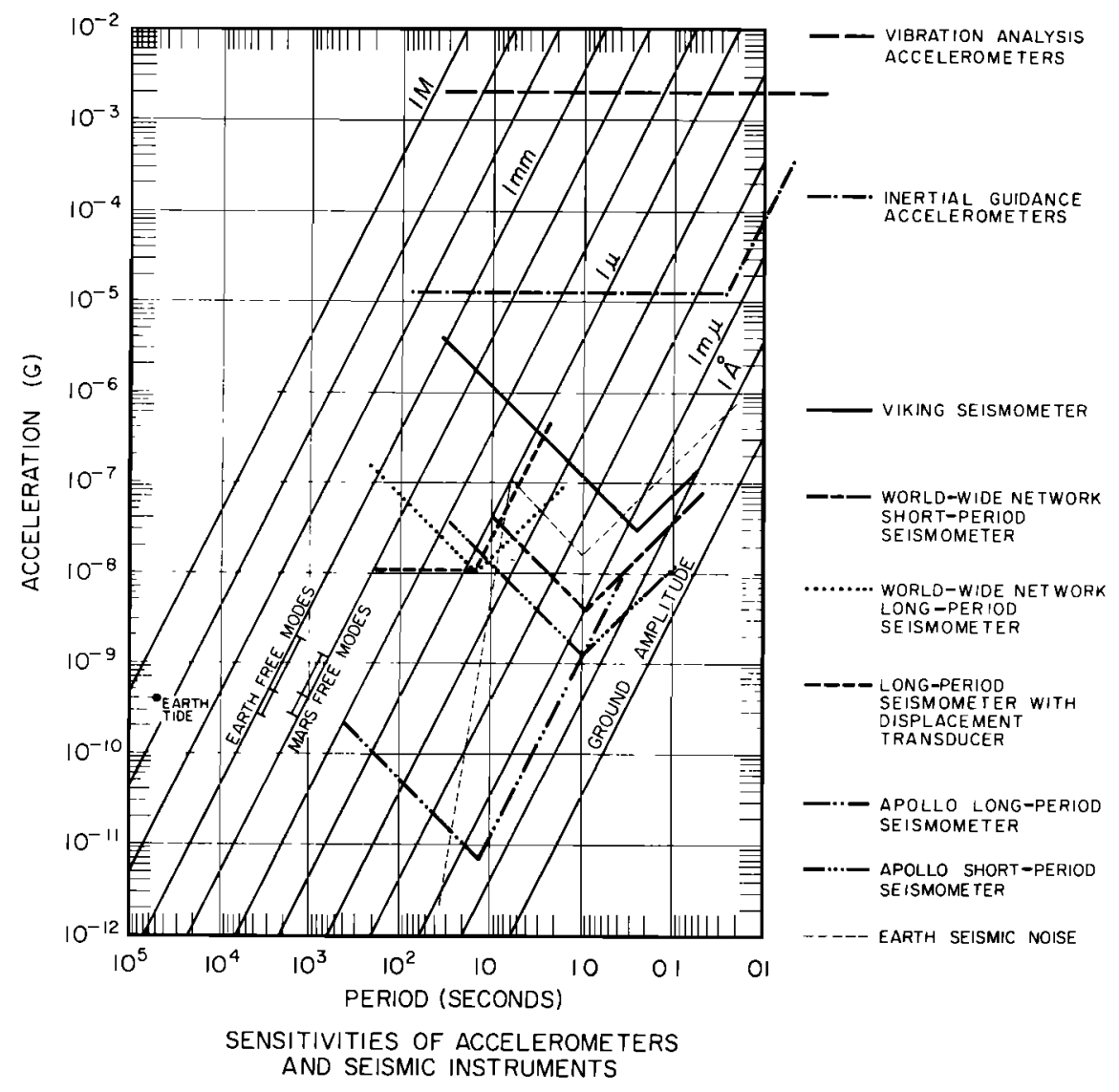

Fig. 7. Comparison of the acceleration sensitivity of the Viking seismometer with the sensitivitues of conventional accelerometers, the U.S. Geological Survey WWSSN, and the Apollo lunar seismometers. The maximum sensitivity of the Viking seismometer is equivalent to that of a relatively quiet short-period seismometer on earth. 


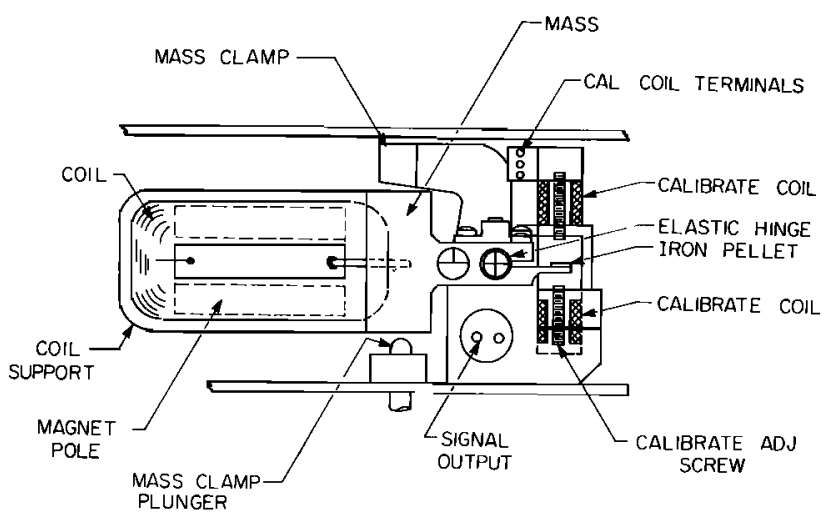

SEISMOMETER SCHEMATIC

Fig. 8. The Viking seismic sensor. As the mass-coil assembly moves in relation to the frame, a signal is induced in the coil as it passes through the field of the magnets. The signal produced is proportional to the velocity of the relative motion.

The instrument may operate in any of three data processing modes.

High data rate mode. Each channel is digitally filtered, and the 7-bit plus sign word is temporarily stored in one of two 2048-bit recirculating memories to await servicing by the lander's data acquisition and processing unit (Dapu). The data rate is 20.2 samples/s/channel.

The filter is a digital implementation of a sixth-order maximally flat (Butterworth) low-pass filter with command-selectable cutoff frequencies of $0.5,1.0,2.0$, and $4 \mathrm{~Hz}$.

Normal mode. The normal mode is the lowest data rate mode, operating at 4.04 samples/min/channel. Its purpose is to investigate the average level and spectral content of the microseismic background. A form of 'comb filtering' is performed by using the digital low-pass filter in conjunction with the frequency response of the inertial sensor. The equivalent noise bandwidth at each filter cutoff is shown in Figure 11. The rectangular response represents an ideal band-pass filter with the same power and peak value as those of the total system frequency response.

After filtering, the absolute value of the data is then passed through a low-pass filter to obtain the 12.7-s running average of the microseismic level which is in turn sampled at the rate of one sample every $14.85 \mathrm{~s}$ on each of the three axes. The digital low-pass filter may be fixed or automatically stepped through each cutoff frequency at the rate of $2 \mathrm{~min}$ per step.

Event or triggered mode. This mode refers to the data compaction mode of operation, where the envelope of the seismic signal and the number of zero crossings rather than the signal itself are sampled at the moderate rate of 1.01 sample/s/axis. To produce the envelope, the absolute value of the seismic signal is smoothed by passing it through the digital filter operating at the $0.5-\mathrm{Hz}$ cutoff frequency. Simultaneously, a running count of the positive axis crossings (a measure of the dominant frequency of the signal) is sampled at the same rate. This combination of sampling the envelope (7-bit word) and axis crossing (5-bit word) results in a 12.3 to 1 reduction in the data required to encode the original signal over the high data rate. The effects of smoothing the envelope, demonstrated by a computer synthesis of its operations, are shown in Figure 12. Recall that the resulting envelope is sampled once per second. Of particular note are the delay in the start of the signal and the rise time, nominally $1 \mathrm{~s}$ each. In addition, there are effects due to transients and incomplete smoothing at frequencies below $0.4 \mathrm{~Hz}$.

The event mode may be initiated by earth command or automatically by enabling an event detector on any one or combination of the three axes. The purpose of the detector is to record seismic events which are transient in nature (Marsquakes or meteor impacts) at the higher data rate of the event mode while it is conserving data by monitoring the seismic
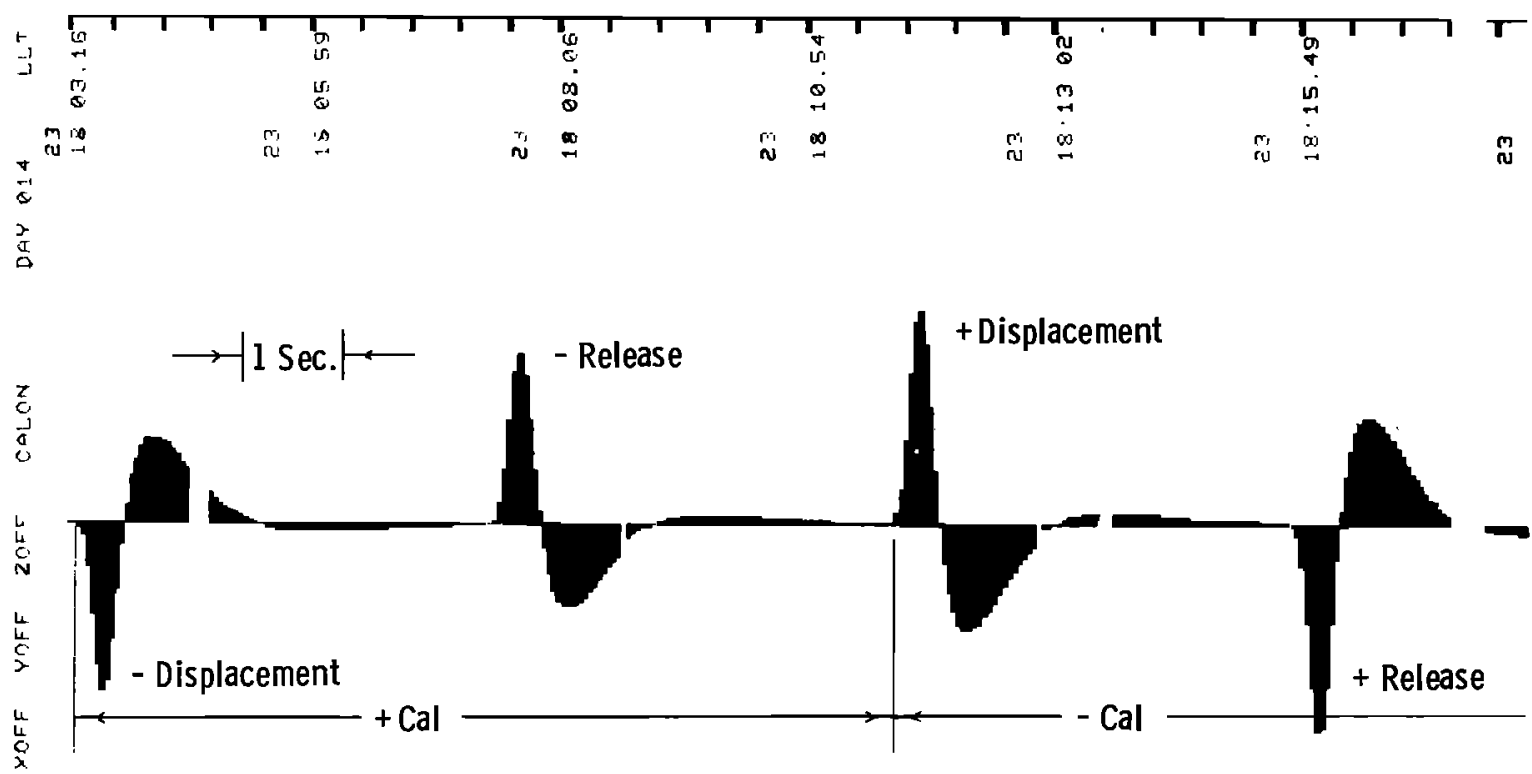

\section{SEISMOMETER CALIBRATION PULSE SEQUENCE}

Fig. 9. Seismometer calibration sequence. The deflection and release of the mass by the calibration coils produce a pair of pulse doublets from which the operational characteristics and the attitude of the seismometer can be ascertained. Calibration sequences are run in the high data rate mode. 


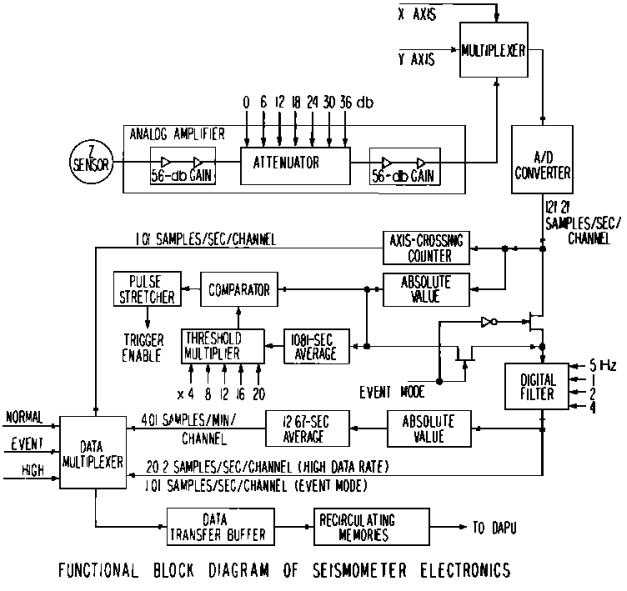

Fig. 10. Block diagram of seismometer electronics. The analog amplifier, shown for the $Z$ axis only, also includes a low-pass filter to prevent aliasing.

background in the normal mode. The trigger level of the detector is a multiple of the long-term (1081 s) average microseismic level. The multiple is selected by ground command from values times $4,8,12,16$, or 20 . When the signal level drops below the average background, the instrument will revert to the normal mode. To reduce the number of data taken from false triggering from lander thermal 'pops,' the event detector on time beyond the normal off time is controlled to be proportional to the time that the level of the event is above the trigger level. This time increase varies from a minimum of $2 \mathrm{~s}$ to a maximum of $1 \mathrm{~min}$ for the longest event.

This autotrigger mode was used during the conjunction period when the total number of Viking science data were limited to those which could be stored on the lander tape recorder during the 44 days of conjunction. This mode is also used in the automatic mission, which is designed to continue lander operations in the event that the ability to load new commands into the lander is lost. The instrument will produce $6170 \mathrm{bits} / \mathrm{h}$ in the normal (or background monitoring) mode, $1.47 \times 10^{5} \mathrm{bits} / \mathrm{h}$ in the event mode, and $1.77 \times 10^{6} \mathrm{bits} / \mathrm{h}$ when it is operating in the high data rate mode.

The instrument contains dual 2048-bit data buffer memories. Two are necessary so that data flow continues while the full buffer is serviced by the lander. The data are in turn transferred along with engineering and scientific data from other experiments to the lander's magnetic tape recorder. Normally, the data are transmitted by the lander to an orbiter for relay to earth; however, they can be transmitted directly to earth at a much lower data rate. The seismometer will transfer 69 buffers per day if it is operating wholly in the normal mode, 1716 buffers per day in the event mode, and 20,700 buffers per day in the high data rate mode. The number of data buffers that the seismometer transfers each day is controlled by the lander and is part of the overall mission planning. It varies from zero to about 3500 buffers per day but is nominally about 1000-1500 buffers per day. The tape recorder on the lander cannot physically hold more than about 18,000 buffers of seismic data.

Normally, the operating time of the instrument is divided among the three modes so as to maximize the amount of time in the event mode with a sampling of high data rate mode during the quietest part of the sol and for calibration. The normal mode is used only to fill in or when other lander activities would produce exceptional lander vibrations. When the data allocation for the seismometer is more than 1700 buffers, the excess data are recorded in the high data rate mode.

\section{INSTRUMENT PeRformanCE}

Following touchdown the azimuths of the horizontal ( $Y$ and $Z$ ) component seismometers were determined with regard to the lander inertial reference system. The positive output from the $Y$ component corresponds to lander motion toward the northwest $\left(\mathrm{N} 31^{\circ} \mathrm{W}\right)$, and the positive output from the $Z$ component corresponds to lander motion toward the southwest $\left(\mathrm{S} 59^{\circ} \mathrm{W}\right)$. The polarity of the vertical $(X)$ component follows the usual convention of positive output for upward displacement. The 'vertical' is $8.2^{\circ}$ from the true vertical toward $\mathrm{N} 82^{\circ} \mathrm{W}$.

The initial calibration pulse amplitudes were in good agreement with predicted values. A gradual increase in calibration pulse amplitudes (18\%) occurred during the first 140 sols, but the relative sensitivities remained closely matched throughout this period (see Figure A-2 of the microfiche appendix). The instrument responses were matched to within approximately $5 \%$ during prelaunch adjustment. The gradual change in pulse amplitudes is a consequence of the decreasing temperature of the lander and thus the lowering of the temperature (resistance) of the transducer and calibration coils. The difference in calibration pulse amplitudes among the three components is caused by the tilt of the instrument.

It was noted early in the experiment that signal amplitudes were consistently smallest on the $Y$ component (10-20\% less than those on $X$ ) and largest on the $Z$ component (20-50\% greater than those on $X$ ) even though the evidence from calibration data showed that the component sensitivities were well matched. In addition, the three components were nearly always in phase, with a high degree of coherence between channels during the brief intervals of high data rate operation in

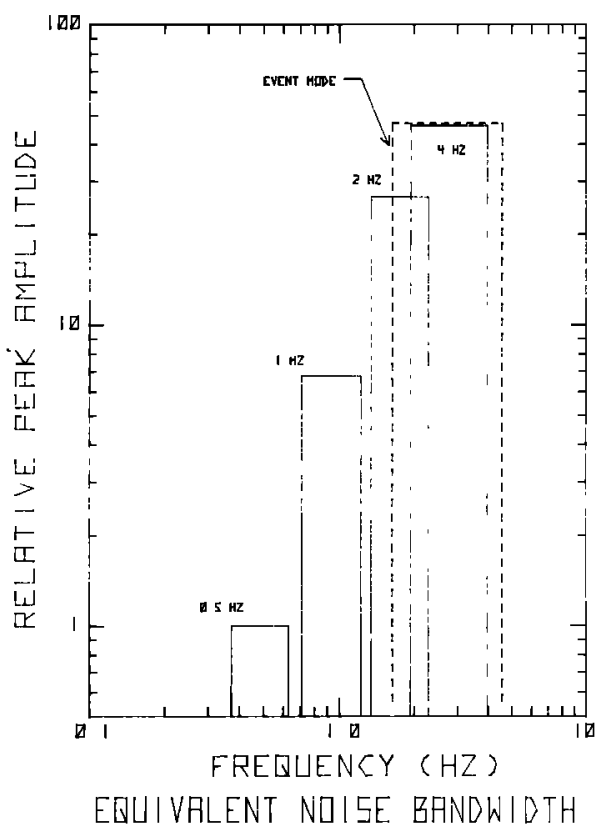

Fig. 11. Equivalent noise bandwidth at each filter setting and of the event mode. The rectangular response represents an ideal bandpass filter with the same area and peak value as the total system frequency response. 

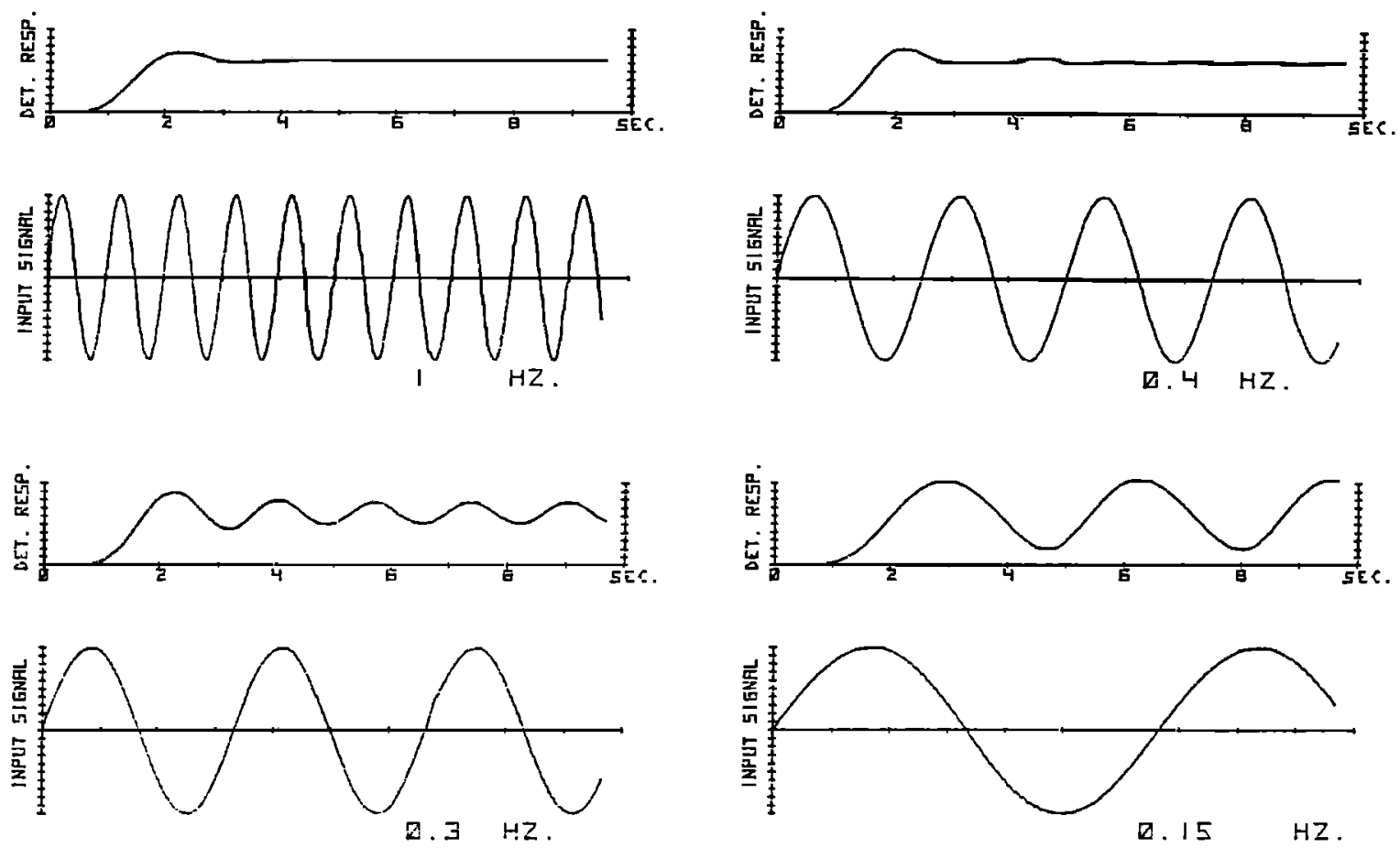

\section{ENVELOPE DETECTOR RESPONSE}

Fig. 12. Event mode envelope detector response. The effects of smoothing low-frequency rectified signals with a $0.5-\mathrm{Hz}$ lowpass filter are shown. The resulting signal is then sampled once per second. The input signals are zero before time zero.

which phase comparisons can be made. This observation can only be explained by rectilinear motion of the lander in a preferred direction. In-phase correlation requires that the preferred direction of motion be up to the west and down to the east, i.e., along the direction of tilt of the lander. This direction is consistent with a rocking motion of the lander about the line connecting footpads 1 and 2 (see Figure A-3). The seismometer is located approximately $1 \mathrm{~m}$ above ground level. The remaining dimensions and angles are shown in Figure A-4. From this the expected amplitude ratios for a rocking motion about the line connecting footpads 1 and 2 are as follows:

$$
A_{x}: A_{y}: A_{z}=1: 0.83: 1.44
$$

These values are in approximate agreement with those observed.

Postlanding imagery revealed that footpad 3 is balanced on a rock. Thus a rocking motion in the direction inferred from seismic data is a reasonable expectation.

A related observation is that wind-induced vibrations of the lander are highest for a given velocity for winds from the east. We would expect that the rocking motion described above would be most easily excited by easterly and westerly winds.

\section{SUMMARY OF OPERATIONS}

On earth, operations such as change of gain and filter setting are usually trivial. During the Viking mission, however, these and other operations (such as change in operating mode and trigger levels) had to be planned many days in advance of the actual execution of the command. This advance planning was necessary to avoid conflicts with other experiments on the lander, to insure the safety of the lander, and because commands could be sent to the lander generally only every other day. During the primary mission, covering the first 62 sols, planning of commands to be sent to the lander was divided into 6-day cycles, each cycle covering three command sequences (uplinks) to be sent to the lander. Planning for each cycle started 16 days before the first command uplink was to occur. As the time for a particular uplink approached, the sequence of commands became less flexible, and more justification was required to incorporate a change. With these restrictions each command could be tested before it was executed on the lander, thus insuring against disastrous errors. During the conjunction period when Mars was behind the sun and totally out of communication with the earth (sols 63-108), the lander operated in a reduced mode, executing commands uplinked before conjunction. During the extended mission (sol 109 to the end of the mission), planning cycles cover 2 weeks, and commands are sent to the lander once per week. During the primary mission, data were returned to earth (downlink) once per day, while downlinks during the extended mission average about twice per week.

The seismometer commands are stored in the lander computer together with the time to be spent in each command. When the end of the command sequence is reached, the sequence is restarted automatically. Uplinks need to be sent only to change a command or command sequence. Each command must specify the mode of operation, vertical gain, horizontal gain, filter frequency, trigger state, trigger threshold, calibration state, and time to be spent in this configuration. In addition, the number of buffers to be recorded and the number of commands to be used can be specified. Until sol 141, two tables of 12 commands each plus a secondary table of three commands could be specified. After sol 141, two tables of 34 entries each became available. 


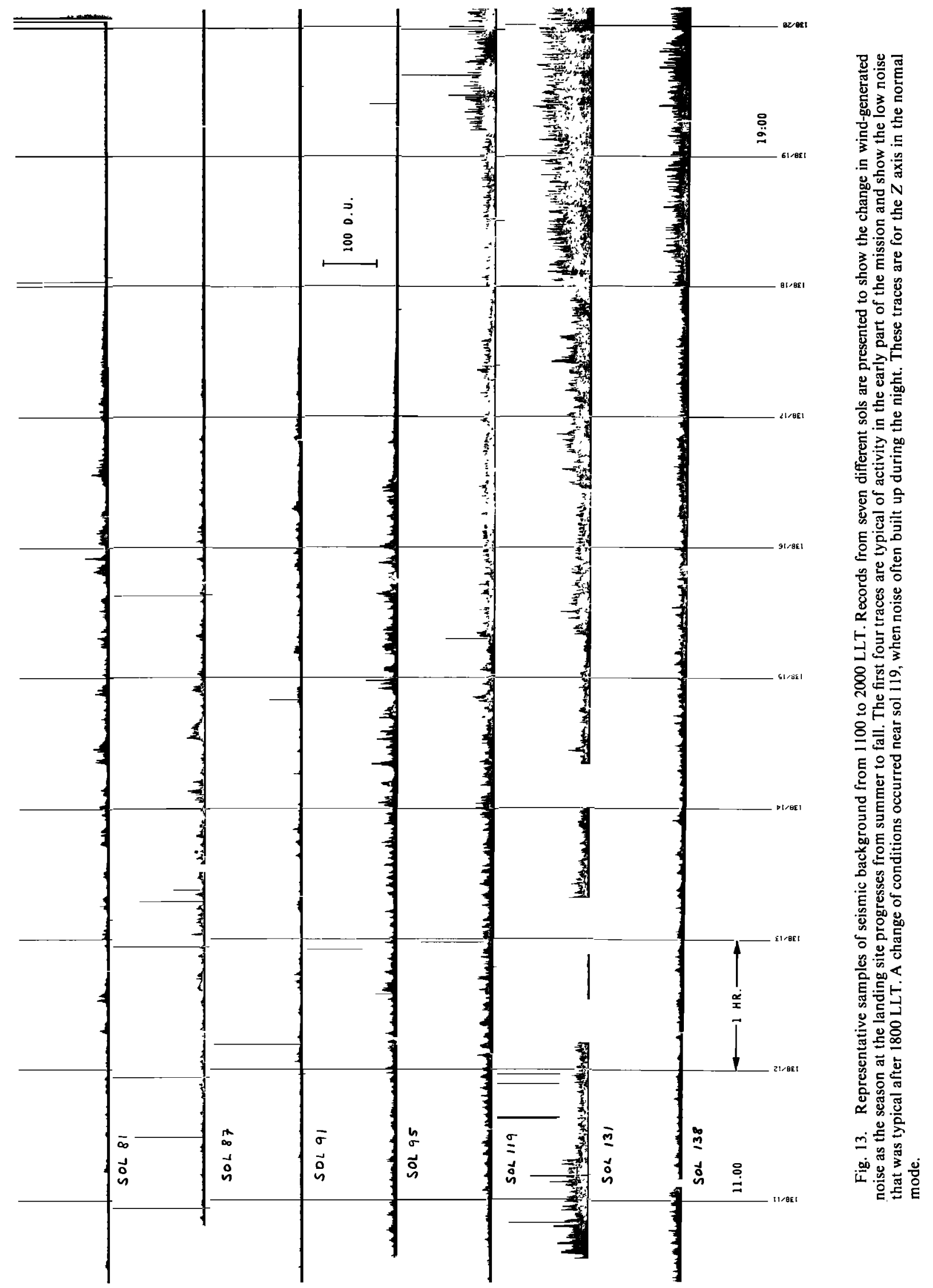


TABLE 1. Examples of Time Lines

\begin{tabular}{|c|c|c|c|c|}
\hline \multicolumn{2}{|c|}{ Period Beginning } & \multicolumn{2}{|c|}{ Period End } & \multirow[b]{2}{*}{ Mode, Other Comments } \\
\hline Sol & Time, LLT & Sol & Time, LLT & \\
\hline \multicolumn{5}{|c|}{ Primary Mission, Sol 17 Downlink } \\
\hline 16 & 0845:48 & 16 & $0947: 39$ & Relay link, no data collected \\
\hline 16 & 0947:39 & 16 & 1053:59 & Event mode \\
\hline 16 & 1053:59 & 16 & $1422: 34$ & Direct link, normal mode \\
\hline 16 & $1422: 34$ & 16 & 2300: 31 & Event mode \\
\hline 16 & $2300: 31$ & 16 & $2317: 31$ & Normal mode, filter stepping \\
\hline 16 & $2317: 31$ & 16 & $2320: 31$ & Calibration, 30-dB attenuation \\
\hline 16 & $2320: 31$ & 16 & $2424: 20$ & High data rate mode, 4.0 filter \\
\hline 16 & $2424: 20$ & 17 & $0843: 16$ & Event mode \\
\hline & & 17 & 0843: 16 & Power off, 2187 buffer dumps \\
\hline \multicolumn{5}{|c|}{ Conjunction, Daily Sequence } \\
\hline$N$ & $0400: 23$ & $N$ & 1900:01 & Normal mode, 4.0 filter \\
\hline$N$ & 1900:01 & $N+1$ & 0300:01 & $\begin{array}{l}\text { Normal mode, } 4.0 \text { filter, } X \text { trigger on, } \\
\text { threshold } \times 12\end{array}$ \\
\hline$N+1$ & 0300:01 & $N+1$ & 0330:01 & Event mode \\
\hline$N+1$ & 0330:01 & $N+1$ & 0330: 17 & Calibration, $30-\mathrm{dB}$ attenuation \\
\hline$N+1$ & $0330: 17$ & $N+1$ & 0359:07 & Event mode \\
\hline & & $N+1$ & 0359:07 & Power off, 152 buffer dumps (see text) \\
\hline \multicolumn{5}{|c|}{ Extended Mission. Sol 129} \\
\hline 129 & 0000:00 & 129 & $0200: 02$ & Event mode \\
\hline 129 & 0200:02 & 129 & $0200: 36$ & Calibration, $30-\mathrm{dB}$ attenuation \\
\hline 129 & $0200: 36$ & 129 & 0700:01 & Normal mode, 4.0 filter \\
\hline 129 & 0700:01 & 129 & 1800:01 & Normal mode, 4.0 filter, $6-\mathrm{dB}$ attenuation \\
\hline 129 & 1800:0I & 129 & 2012:31 & Normal mode, 4.0 filter \\
\hline 129 & 2012:31 & 130 & 0000:00 & Event mode, 500 buffer dumps \\
\hline \multicolumn{5}{|c|}{ Extended Mission, Sol 156} \\
\hline 156 & 0000:00 & 156 & $0159: 48$ & Event mode \\
\hline 156 & $0159: 48$ & 156 & 0200: 18 & Calibration, 30-dB attenuation \\
\hline 156 & $0200: 18$ & 157 & 0000:00 & Event mode, 1720 buffer dumps \\
\hline
\end{tabular}

Horizontal and vertical attenuations are the same and are $0 \mathrm{~dB}$ unless they are otherwise noted. No filter means that the low-pass filter was set to $0.5-\mathrm{Hz}$ cutoff.

The planning procedures and structure for the lander operation have been described by Lee [1976]. Readers are referred to this paper for a more complete description of the uplink procedures. Although a large amount of time was spent by each team in these planning sessions, the necessity of the system cannot be denied.

Before touchdown the commands loaded into the tables were to survey the ambient seismic noise at various gain levels, with approximately 500 buffers of data allocated per sol. The data returned from this phase of the operation demonstrated that the seismometer could be operated at all times at maximum gain. After sol 9, high enough data rates were available to use the event mode most of the time. During conjunction, 152 buffers per sol were available to the seismometer. Only 1 hour of event mode per sol was scheduled with 8.5 hours of the remaining 23.5 hours of normal mode set aside for triggerenabled operation. If the trigger was not activated, 141 buffers would be taken; thus 11 buffers were set aside for triggered event mode. In the extended mission a more flexible schedule is followed. On occasion, 1700 buffers are available, continuous event mode recording thus being permitted. At other times, fewer data, down to 500 buffers per sol, were available.

To date, the seismometer has been operated nearly continuously except for two periods: sols 103 and 104 and sols 141-146. One hundred and seventy-one sols of data have been collected, consisting of 87 sols of normal mode data, 75 sols of event mode data, and 63 hours of high data rate mode data (the balance is lost time due to playback periods of the lander tape recorder for relay to the orbiter). Some detailed examples of data collection are given in Table 1. Periods when the seismometer was inhibited by other experiments have been ignored except for inhibitions due to the relay link and direct link, since these occurred on a regular basis during the primary mission and were generally of short duration.

\section{LANDER NoISES}

Since the seismometer is located on top of the lander, it is important to understand all internal sources of noise, the response of the lander to wind, and the effect of the lander on external seismic signals. A catalog of these noise sources and representative signatures of each are given in the microfiche appendix to this report. Noises generated inside the lander are generally of short duration, infrequent, and distinctive in nature and occur at predictable times. There is therefore little likelihood that they would be confused with natural events, but they do complicate data processing, and it has taken considerable effort to identify and catalog them. The internal sources identified are tape drive, $X$ ray, sample dump, antenna tracking, camera motions, operations of the soil sampler, and electrical transients from other experiments. The known vibrational frequencies of these activities are in agreement with the frequencies measured by the seismometer. These are generally greater than $4.7 \mathrm{~Hz}$. When the soil sampler arm is in the extended configuration, frequencies of $2-4 \mathrm{~Hz}$ are observed, the dominant frequency depending on the extension. The $Q$ of the arm is about 200. Wind-induced vibrations of the lander are also more pronounced when the arm is extended. Fortunately, the arm is usually stowed. Before sample delivery the collector head is usually vibrated at 8.8 or $4.4 \mathrm{~Hz}$ for a short period of time. These frequencies are clearly observed by the seismometer at the appropriate times.

There is no firm evidence that any high $Q$ lander resonances 


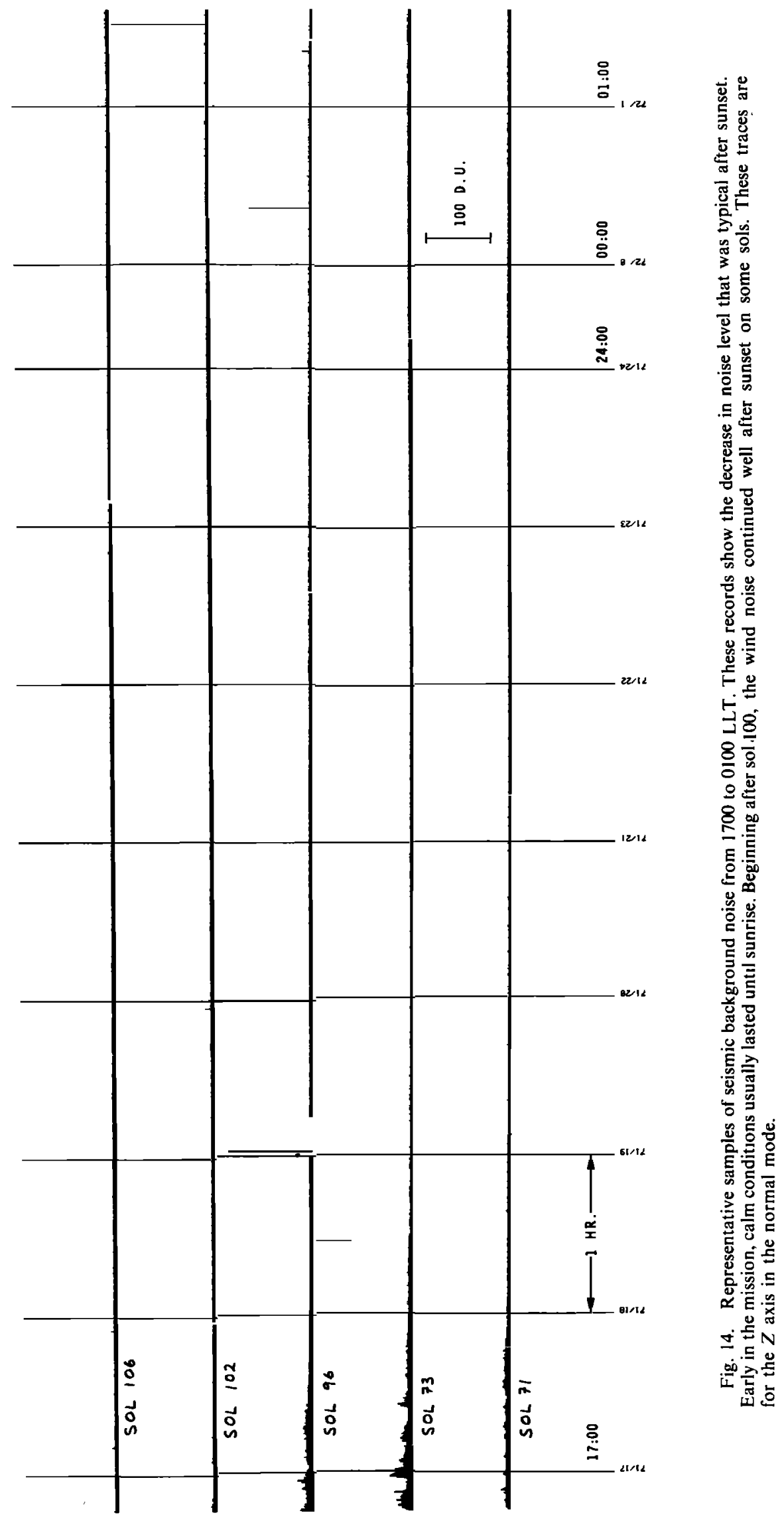




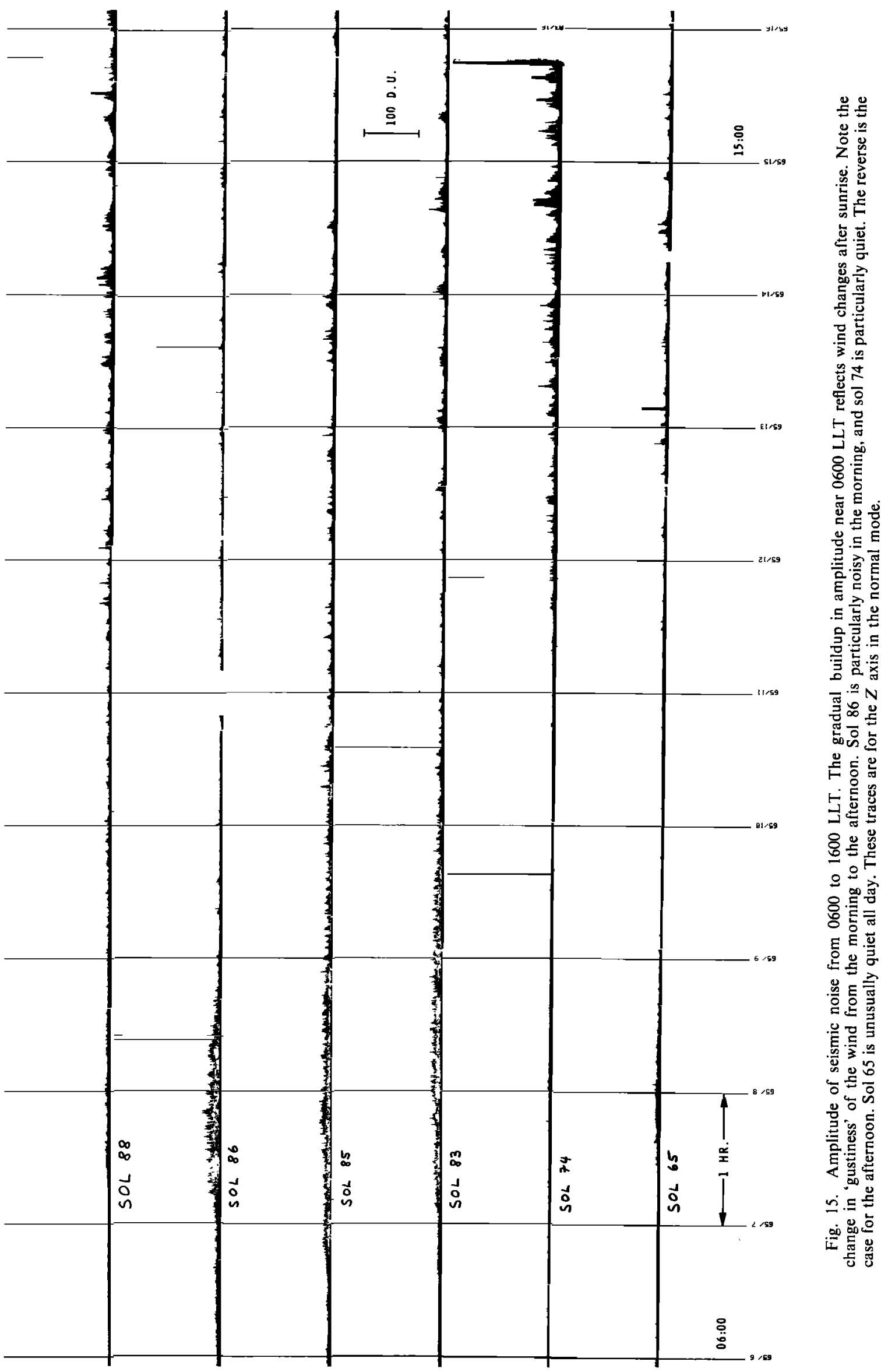




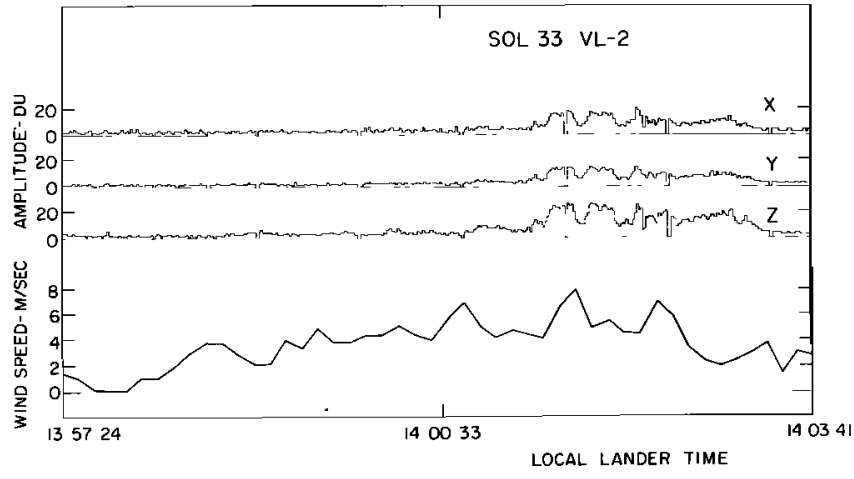

Fig. 16. Correlation of wind speed with seismic noise on sol 33. In this case, wind gusts of 7-8 m/s produced noise levels of $16-20 \mathrm{du}$. The wind detection threshold for seismic noise is about 3-4 m/s.

are excited by any lander activity. There is a suggestion of a resonance at $7.6 \mathrm{~Hz}$, seen during the initial slew of the highgain antennae and occasionally during a sampling arm operation, and also a resonance at frequencies of $14-15 \mathrm{~Hz}$ based on infrequent high-amplitude impulsive events of possible thermal origin. High $Q$ lander resonances were designed to be outside the useful frequency band of the seismometer. There is also no evidence for high $Q$ wind-induced lander resonances.

\section{Wind-Generated NoISE}

The main source of external noise is the wind blowing on the lander. Generally speaking, the wind noise background repeats from day to day, but the background is quite variable when it is looked at in detail. Some days are very noisy (windy), and some days are very calm. Martian winds and hence the background noise level vary distinctly with the season. Prior to conjunction, during summer in the northern hemisphere (sols 0-60), the average wind speeds were observed to be somewhat higher in the morning than in the afternoon, but individual wind gusts had higher peak speeds in the afternoon [Hess et al., 1977]. This is reflected in the seismic data (Figure 13). The highest seismic background typically occurred between 1300 and 1700 hours (Mars local time), when the seismic amplitudes ranged from 10 to 40 digital units (du). Noise bursts were typically $1-3 \mathrm{~min}$ in duration, separated by time intervals of 10-50 min. The seismic background level drops between individual bursts of wind; this drop indicates that there is little direct transfer of energy into the ground by the wind and that the principal effect on the seismometer is produced by wind-induced vibrations of the lander.

Typically, the quietest recording interval occurs from about 1800 hours local lander time (LLT) ( 2 hours before sunset) to about 0400 hours the following morning (Figure 14). During this time interval the wind speed decreases to less than 1-2 $\mathrm{m} / \mathrm{s}$, and the seismic noise background level falls to between $\mathrm{I}$ and $2 \mathrm{du}$. (In the event mode at maximum sensitivity, $1 \mathrm{du}$ corresponds to $2 \mathrm{~nm}$ of instrument displacement at $3 \mathrm{~Hz}$.) Around Martian sunrise the background noise level generally increases to about 2-3 du (Figure 15).

An example of the correlation between seismic noise activity in the event mode and a wind gust recorded on sol 33 is shown in Figure 16. In this case a $7-$ to $8-\mathrm{m} / \mathrm{s}$ wind gust produced a seismic signal of 16- to 20-du amplitude. A similar figure is shown in the microfiche appendix (Figure A-18) covering a different period of time. The threshold of wind detectability of the seismometer, as seen in the event mode at maximum sensitivity, appears to be about $3-4 \mathrm{~m} / \mathrm{s}$.
Figure 17 shows the high degree of correlation of the amplitude of the seismic signals recorded by the seismometer in the normal mode ( $Z$ component) with the wind speeds recorded by the meteorology experiment. The data shown in Figure 17 indicate that the seismic amplitude is proportional to the square of the wind speed, as is expected for turbulent flow. Detailed correlations are difficult, since the seismic data represent averages and the wind data are widely spaced point samples. A similar figure for $X$ component data in the event mode is shown in Figure A-19. In the high data rate mode of operation, where no averaging is involved, seismic activity peaks and wind speed peaks are also reasonably well correlated. Figure A-20 shows the correlation between $Z$ component amplitudes and wind speeds in the high data rate mode on sols 35 and 36. Data observed in the high data rate mode also show that the seismic amplitude increases as the square of the wind speed. Data plotted in Figure 17 also show the correlation of seismic noise for different wind directions. The data are in good agreement with the relation $A=0.15 V^{2}$, where $A$ is the mean seismic amplitude in digital units (event mode) and $V$ is the mean wind speed in meters per second. The scatter of data at low speeds suggests a threshold of about $3 \mathrm{~m} / \mathrm{s}$.

During the period of conjunction (sols 60-90) the background noise level was extremely low from 1800 hours each evening to 0700 hours the following morning, the background level increasing slightly starting between 0630 and 0700 hours. The afternoon gusty wind period began between 1018 and 1230 hours each day, usually close to local noon. Peak gusts usually occurred near 1430 and 1520 hours. Typically, 8-12 wind gusts were observed between 1200 and 1800 hours lasting $8-15 \mathrm{~min}$ and separated by intervals of roughly $40 \mathrm{~min}$. The gusts produced seismic amplitudes ranging from 10 to $20 \mathrm{du}$ in the normal mode.

Northwest winds typically occur in the afternoon from 1300 to 1800 hours. Northeast winds generally occur in the early morning hours, changing to southeast winds from 0700 to 0900 hours and to southwest winds from 0900 to 1200 hours [Hess et al., 1977]. During the postconjunction period from sols 110 to 118 the winds were generally less than the seismic threshold $(\sim 3 \mathrm{~m} / \mathrm{s})$ from about 1800 hours to sometime be-

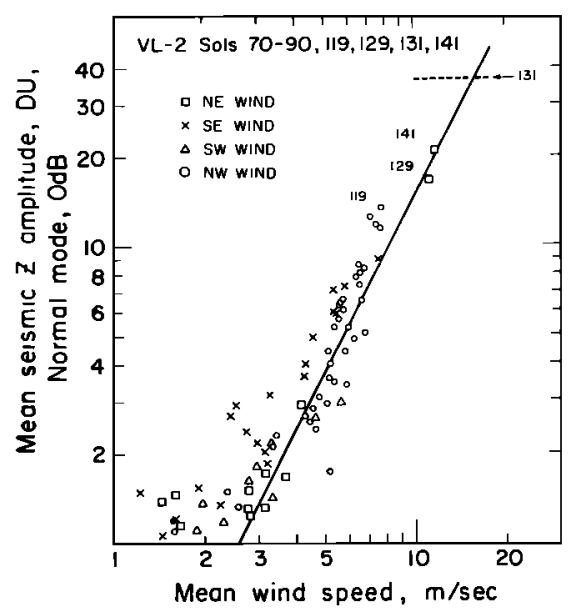

Fig. 17. Correlation between wind speed and normal mode seismic amplitude ( $Z$ component) for winds from different directions. The solid line corresponds to the slope expected if seismic amplitude is proportional to the square of the wind speed, as is appropriate for turbulent flow. The scatter of points below wind speeds of $3 \mathrm{~m} / \mathrm{s}$ is due to noise from other sources than wind. 


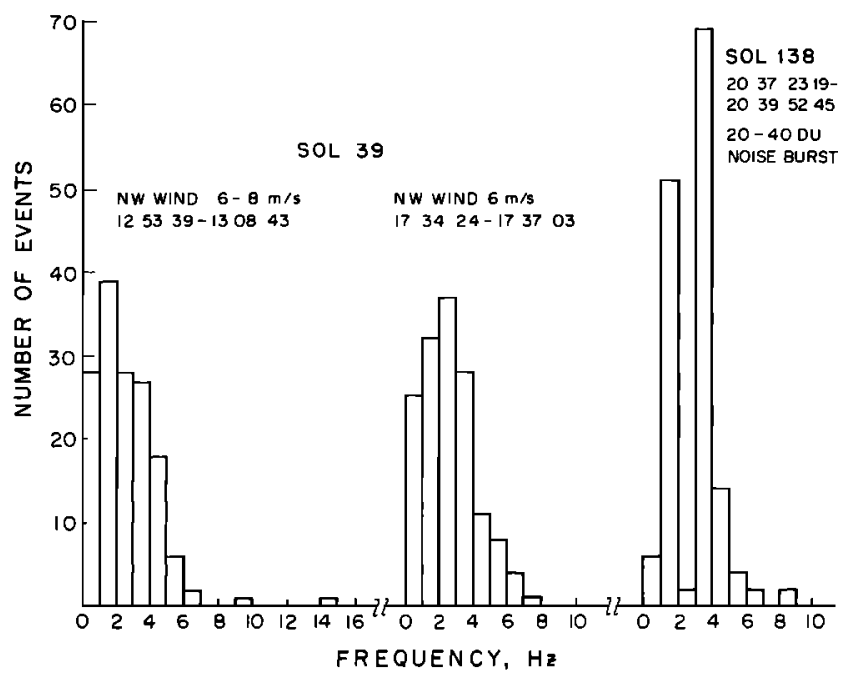

Fig. 18. Axis-crossing histograms for windy periods. The number of events (ordinate) is a measure of the amplitude at the corresponding frequency. If high $Q$ lander resonances were excited by winds, these histograms would show sharp peaks at the resonant frequencies. Such peaks are not observed, and the shape of the histograms reflects the frequency response of the seismometer.

tween 0600 and 1030 hours LLT. Winds generally averaged from 2.5 to $4.9 \mathrm{~m} / \mathrm{s}$ from 1000 to 1800 hours LLT, with maximum wind peaks from 6.4 to $11.5 \mathrm{~m} / \mathrm{s}$. The seismic data were characterized by quiet nights, relatively quiet mornings, and increased activity in the afternoon.

Unusually high seismic background was recorded on sol 119 beginning at 1100 hours LLT. The noise level was continuous and unlike the usual afternoon windy periods. Average wind speeds in this period were unusually high even after 1800 hours, when it is usually quiet. The wind speeds averaged greater than $7.5 \mathrm{~m} / \mathrm{s}$ during the time interval from 1200 to 1800 hours, with peak gusts of $11.6,13.2$, and $15.1 \mathrm{~m} / \mathrm{s}$. Quite surprisingly, the wind speeds from 1800 hours to midnight ranged from 4.7 to $6.4 \mathrm{~m} / \mathrm{s}$ for successive $1 \frac{1}{2}$-hour-long meteorology samples. Wind speed peaks of $11.4,10.7,9.5$, and 8.9 $\mathrm{m} / \mathrm{s}$ were observed throughout this normally quiet period.

Continuous, although lower-level, activity also occurred in the early morning hours on sols 118, 121, 128, 129, and 130. Exceptionally high activity was observed on sol 131 lasting until 0200 hours LLT on sol 132. The mean seismic $Z$ amplitude was 37.1 du with a maximum of $56 \mathrm{du}$ in the normal mode at $0 \mathrm{~dB}$. Extrapolating the relation found earlier (Figure 18) suggests mean wind speeds of $16-18 \mathrm{~m} / \mathrm{s}$, a value higher than any previously reported. Unfortunately, because of a temporary malfunction, there were no meteorology data during this period. Imagery results show that at some time between sols 110 and 120 the optical depth apparently increased by an order of magnitude. Average temperatures were also observed to drop rapidly on sols 119 and 120,128 , and 131. All of these observations are suggestive of an unusual meteorological disturbance or the onset of a new meteorological regime due to the change of seasons.

Before landing, there was some question about whether the wind would excite high $Q$ resonances in the lander. To examine this question, zero-crossing (frequency) histograms for different times of day and wind conditions were compiled. Figure 18 shows a zero-crossing histogram for a moderate northwesterly wind during sol 39 at two times during the afternoon windy period. The predominant frequency is be- tween 2 and $3 \mathrm{~Hz}$, close to the natural period of the seismometer system. Also shown are data from a noise burst on sol 138 . Figures A-21 and A-22 show histograms compiled for a 15min interval during an unusually windy period near Martian midnight on sol 133 and a similar 5-min interval on sol 139. As before, the main frequency appears to be concentrated between 2 and $3 \mathrm{~Hz}$. There are no obvious lander resonances or preferred excitation frequencies. The shape of the histograms reflects the response of the instrument.

\section{Wind STRESSES ON THE LANDER}

Wind stresses on the lander are of the order of 0.002-0.2 $\mathrm{dyn} / \mathrm{cm}^{2}$, depending on wind velocity. Although the wind gusts themselves are of generally low frequency, large changes in amplitude occur of the order of seconds or less. The lander itself can introduce a high-frequency motion due to turbulent shedding at preferred frequencies. The latter are related to wind velocity, the characteristic dimensions of lander components, and the Strouhal number via

$$
f=s V / h
$$

where $V$ is the wind velocity, $h$ is the dimension of the lander or extended components, and $s$ is the Strouhal number, which is about 0.2 for cylindrical or blunt obstacles. Taking $1 \mathrm{~m}$ as the characteristic dimension of the lander, the shedding frequency is $4 \mathrm{~Hz}$ for $20-\mathrm{m} / \mathrm{s}$ winds and $0.4 \mathrm{~Hz}$ for $2-\mathrm{m} / \mathrm{s}$ winds. Therefore vortex shedding frequencies are in the seismic band.

There is a slight indication from the seismic data that increased frequency correlates with increased amplitude (and wind velocity), but the passband of the instrument is too narrow to make a detailed correlation at this stage in the data processing.

The vibrations of the lander due to wind action are proportional to the square of the wind velocity $V$. Wind forces $\tau$ on the lander can be written

$$
\tau \sim \rho A V^{2}
$$

where $A$ is the cross-sectional area of the lander and $\rho$ is the density of the atmosphere. The seismometer threshold to winds is about $3 \mathrm{~m} / \mathrm{s}$. The location of the seismometer on the top of the lander magnifies the wind-induced strains, and the response of the lander to wind stresses can be written

$$
\tau=M \epsilon=M d / l
$$

where $M$ is the effective modulus of the lander system, $\epsilon$ is the strain, $d$ is the displacement, and $l$ is the distance of the seismometer from the contact between the legs and the surface. In order to reduce wind noise therefore, an optimum seismic experiment would minimize $A$ and $l$ and maximize $M$. The cross-sectional area of the lander is about $10^{4} \mathrm{~cm}^{2}$. For an offloaded seismometer this could be reduced to $10^{2} \mathrm{~cm}^{2}$, an immediate decrease in wind stresses of a factor of $10^{2}$ being given, raising the threshold wind speed to $30 \mathrm{~m} / \mathrm{s}$. The modulus $M$ of a compact surface instrument could probably be increased by about an order of magnitude, and the height of the package could be decreased by an order of magnitude, an improvement of another 2 orders of magnitude thus resulting in the signal-to-noise ratio. In principle therefore a $10^{4} \mathrm{im}$ provement in seismometer sensitivity could be usefully employed for a Martian seismometer, and direct wind-induced noise would be no problem for winds as high as $300 \mathrm{~m} / \mathrm{s}$. Future missions to Mars could have seismometers of Apollolike sensitivity. This is important not only to increase the range 


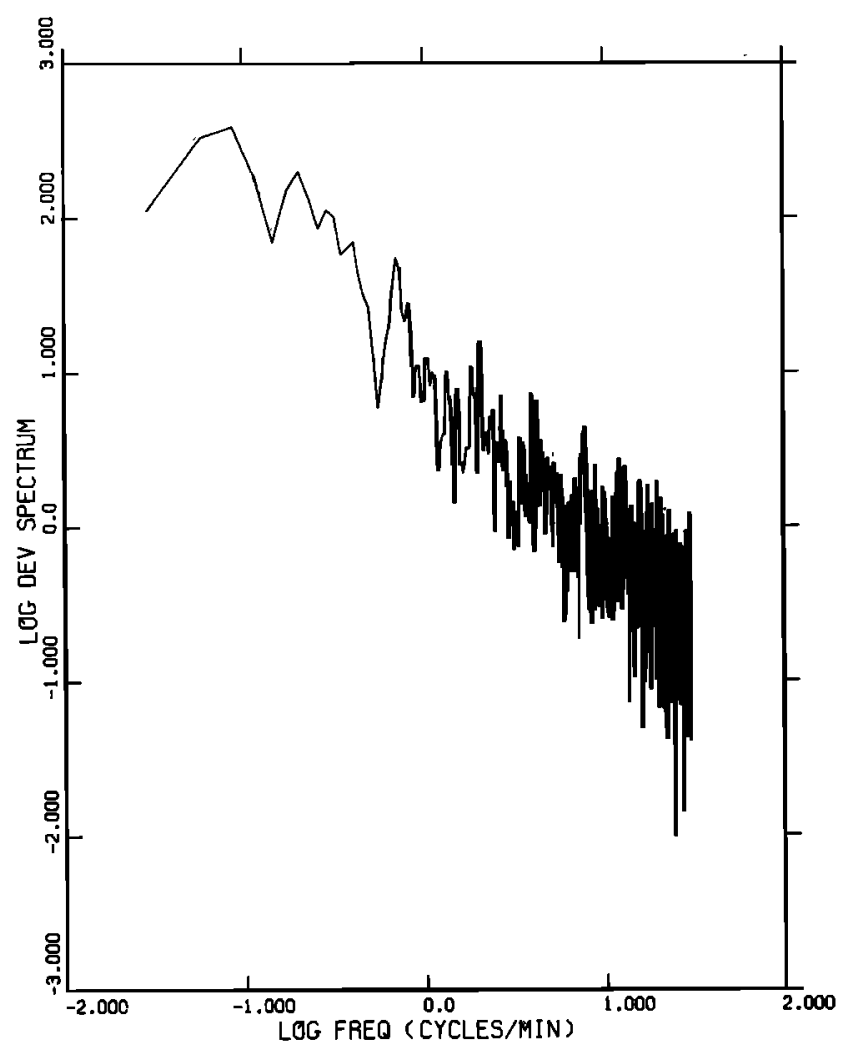

Fig. 19. Power spectrum of event mode amplitude envelope data for a 21 -min period starting at $1429: 18$ on sol 16. Two major wind gusts during this period provide the energy for this spectrum. The slope of the spectrum at frequencies higher than 1 cycle per minute is about -0.9 .

of detectability but because the number of small events is undoubtedly much greater than the number of large events. Magnitude-frequency relationships indicate that for each order of magnitude increase in instrument sensitivity, there will be an order of magnitude increase in number of detected events. An optimal seismic network on Mars therefore could usefully employ magnitude zero events to define the seismicity and internal structure of the planet.

\section{Noise Spectra}

The spectra of wind-induced seismic noise are not only useful for micrometeorological purposes but are potentially useful discriminants for identifying natural events. Most of the meteorological data have been taken at sampling intervals of $4 \mathrm{~s}$ or greater, although occasionally wind speed is sampled at 1.2-s intervals. Higher sampling is necessary to define the turbulent characteristics of the lower Martian atmosphere.

Figure 19 shows the power spectrum of the amplitude envelope for a 21.5 -min section of event mode data ( $Z$ axis) starting at 1429:18 hours on sol 16. There are two major wind gusts in this period. At periods of less than $1 \mathrm{~min}$ the power spectrum has a mean slope of -0.9 . Data at periods of less than $15 \mathrm{~s}$ may be aliased by high-frequency changes in the envelope and should be ignored.

Figure A-23 gives the power spectra of all three axes of the seismometer for a high-wind period of sol 131 starting at 2015: 34 hours and extending for $55 \mathrm{~min}$. The nearly monotonic decrease of power with increasing frequency with a slope slightly less than unity is typical of the wind noise spectra on Mars.
Table 2 summarizes the spectral slopes for 34 samples of wind noise. The slopes are substantially lower for the vertical component than they are for the horizontal. The slopes vary from 0.58 to 0.98 with a mean of $0.91 \pm 0.10$ on the $Z$ axis.

\section{Transient Events}

Many transient seismic signals have been detected by the Viking seismometer. However, as is described elsewhere in this paper, wind-induced vibrations of the lander greatly complicate the problem of establishing the sources of the observed signals. Winds were light during the midsummer to late summer period of operation at site 2; thus about half of each day was left relatively free of wind disturbances. With the approach of fall at site 2 , however, winds began to increase to the point where wind disturbances occasionally persisted throughout the Martian day. In the absence of additional stations for intercomparison our only recourse is to search the records for signals of unusual character and to determine if these signals correlate with local-wind-generated or lander-generated events. We have seen that seismic signals do correlate quite closely with variations in wind speed and direction and that the patterns of these variations can be quite complex. Onsets of wind gusts can be abrupt, and isolated gusts can occur during otherwise quiet periods. Unfortunately, wind data are not obtained by the meteorology experiment on a continuous basis, so correlation with seismic data is not always possible. For example, during the conjunction period, wind data were only available for about $17 \%$ of the total time.

From the seismic data available through sol 144, eight signals were selected for further analysis prior to receipt of wind data. These are shown in Figures A-24 to A-29 and Figures 20 and 21 . Selection was based primarily on their relatively impulsive onsets and relatively low axis-crossing counts. Following receipt of wind data, six of these events were found to correlate with wind gusts. Wind data were not obtained during the time intervals of the remaining two signals. The times, maximum winds, and average signal frequencies for each event are given in Table 3. Signal frequencies are average values for the vertical component over 40 -s samples centered at the time of peak signal amplitudes. On the basis of these data, therf appears to be some tendency toward increasing signal fre-

TABLE 2. Seismic Noise (Wind) Spectra, 55-Min Samples, Frequency Range $0.05-0.5 \mathrm{~Hz}$, and PSD With Mean Removed

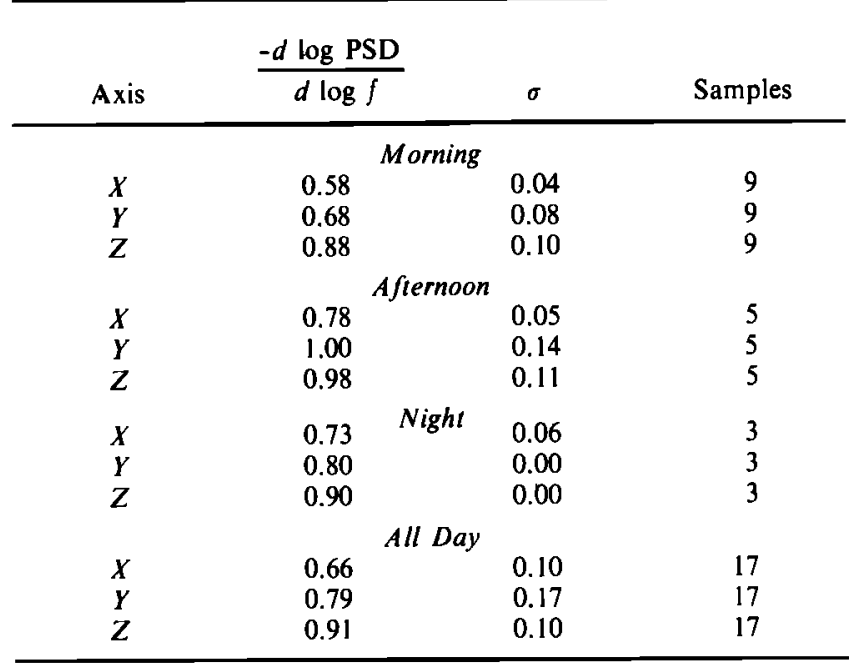

PSD is power spectral density. 


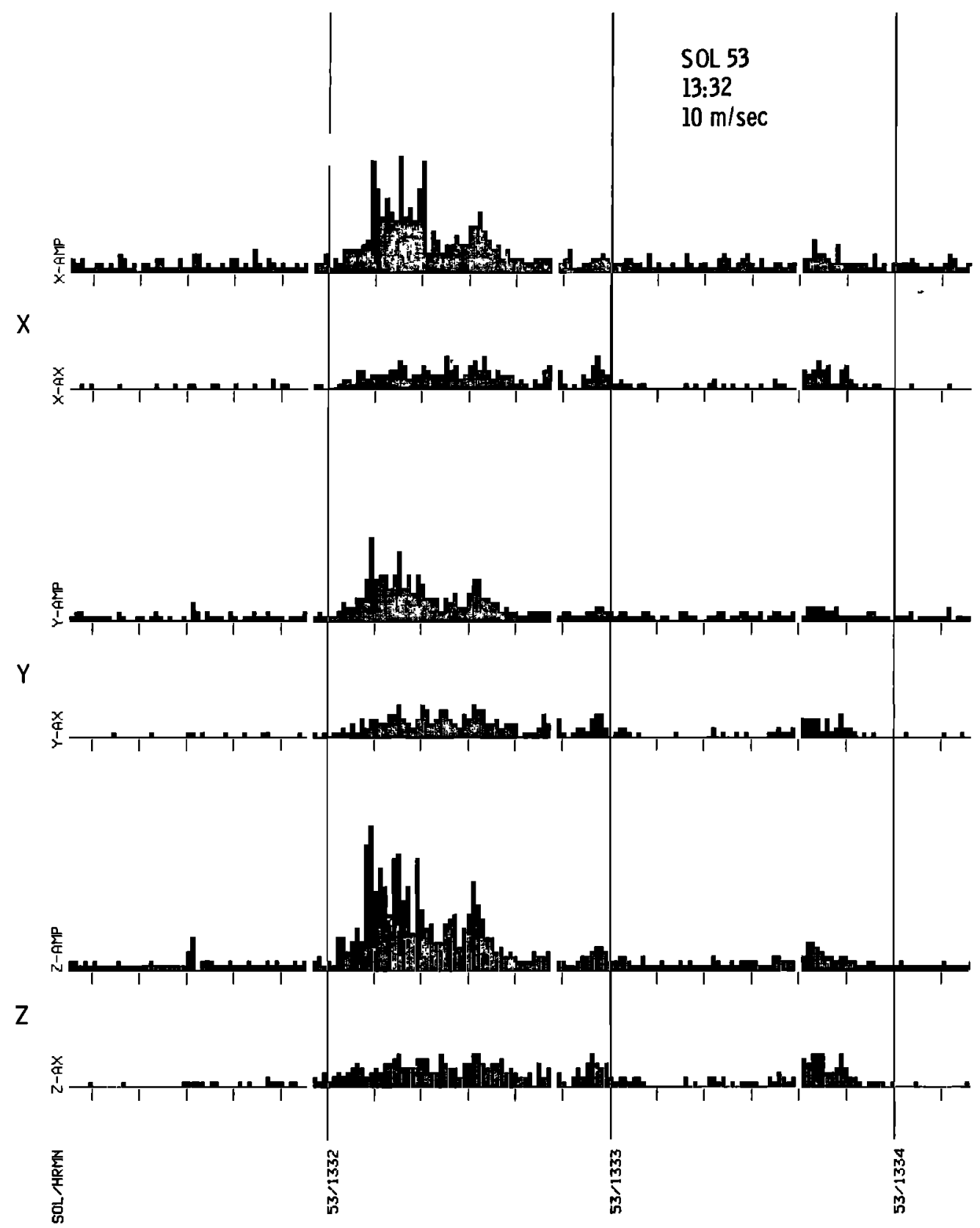

Fig. 20. Sol 531332 LLT event recorded in event mode. Although it was thought to be a possible Marsquake when it was first observed, this event was later correlated with a $10-\mathrm{m} / \mathrm{s}$ gust of wind.

quency with increasing wind speed. However, the frequency content of the sol 80 signal, for which there are no wind data, is unusually high in relation to the remainder of the data set. Other unusual characteristics of the sol 80 signal are worthy of note: (1) It is the only signal of the group that occurred during the normally quiet period of the early morning, (2) both amplitude and frequency variations occur at intervals of about $10 \mathrm{~s}$, and (3) the amplitude variations are similar to those of two local events recorded by the Viking seismometers during operational tests in southern California. Regarding the first point an isolated wind gust was detected during the late evening hours of sol 82 , so such gusts, though they are infrequent, can occur.

The 10-s periodicity in the amplitude variation of the sol 80 signal is clearly seen in the spectrum of the signal envelope in Figure 22. A pronounced spectral peak of $10 \mathrm{~s}$ is not seen in the spectra of any of the other candidate signals except that of the sol 42 signal. The signal character in the time domain is quite different in these two cases, however. The high-frequency character of the sol 80 and sol 42 signals is also distinctly different. Histograms giving the distribution of zero crossings for the sol 80 event and three wind events are given in Figure 23. The dominant frequency on the horizontal axes is $3-4 \mathrm{~Hz}$ in all cases, close to the natural period of the seismometer system. The same is true for the vertical component of the known wind events. The vertical $(X)$ component for the sol 80 event has peaks at 2, 4, and $7 \mathrm{~Hz}$. A peak at 6 or $7 \mathrm{~Hz}$ also shows up in the horizontal components of the wind gusts. The sol 80 event is distinctly bimodal in comparison to the other events.

The sol 80 signal is compared in Figure 24 with two terrestrial signals recorded with a test instrument operated at the 
California Institute of Technology campus in Pasadena. The uppermost signal is an aftershock of the 1971 San Fernando earthquake, recorded at a range of $65 \mathrm{~km}$, the middle signal was generated by 102 metric tons of chemical explosive detonated in a rock quarry at a distance of $69 \mathrm{~km}$, and the bottom signal is the sol 80 event recorded on Mars.

Obviously, a strong case for seismic origin for any Martian signal cannot be made until a strong signal is obtained at a time when wind speeds are known to be low. However, on the basis of the similarities between the signals recorded from local shallow sources in southern California and the sol 80 signal, let us see if the amplitude-duration relations are consistent with the hypothesis that the sol 80 signal was generated by a Marsquake similar in crustal structure to that of the terrestrial examples. Interpreting the amplitude and frequency variations as $P$ and $S$ arrivals, as shown in Figure 25, the $S$-P time is approximately $13 \mathrm{~s}$. This places the epicentral distance at about $110 \mathrm{~km}$ for a surface focus event if crustal velocities normal for the earth are assumed. The maximum amplitude of the shear wave arrival (peak 1, average of $Y$ and $Z$ components) is $188 \mathrm{~nm}$ at a frequency of about $5 \mathrm{~Hz}$. The trace amplitude on a standard Wood-Anderson seismograph $\left(T_{0}\right.$ of $0.8 \mathrm{~s}$ and magnification of 2800 ) would be $0.53 \mathrm{~mm}$, yielding a Richter magnitude of 2.8 for the event.

The magnitude-duration relation determined for southern California is given by

$$
M_{t}=-0.68+1.75 \log t+0.0014 \Delta
$$

where $t$ is the signal duration in seconds and $\Delta$ is the distance in kilometers. If $t=70 \mathrm{~s}$ and $\Delta=110 \mathrm{~km}$ are used, this expression yields $M_{t}=2.7$, in good agreement with the magnitude estimate from maximum signal amplitude. The duration

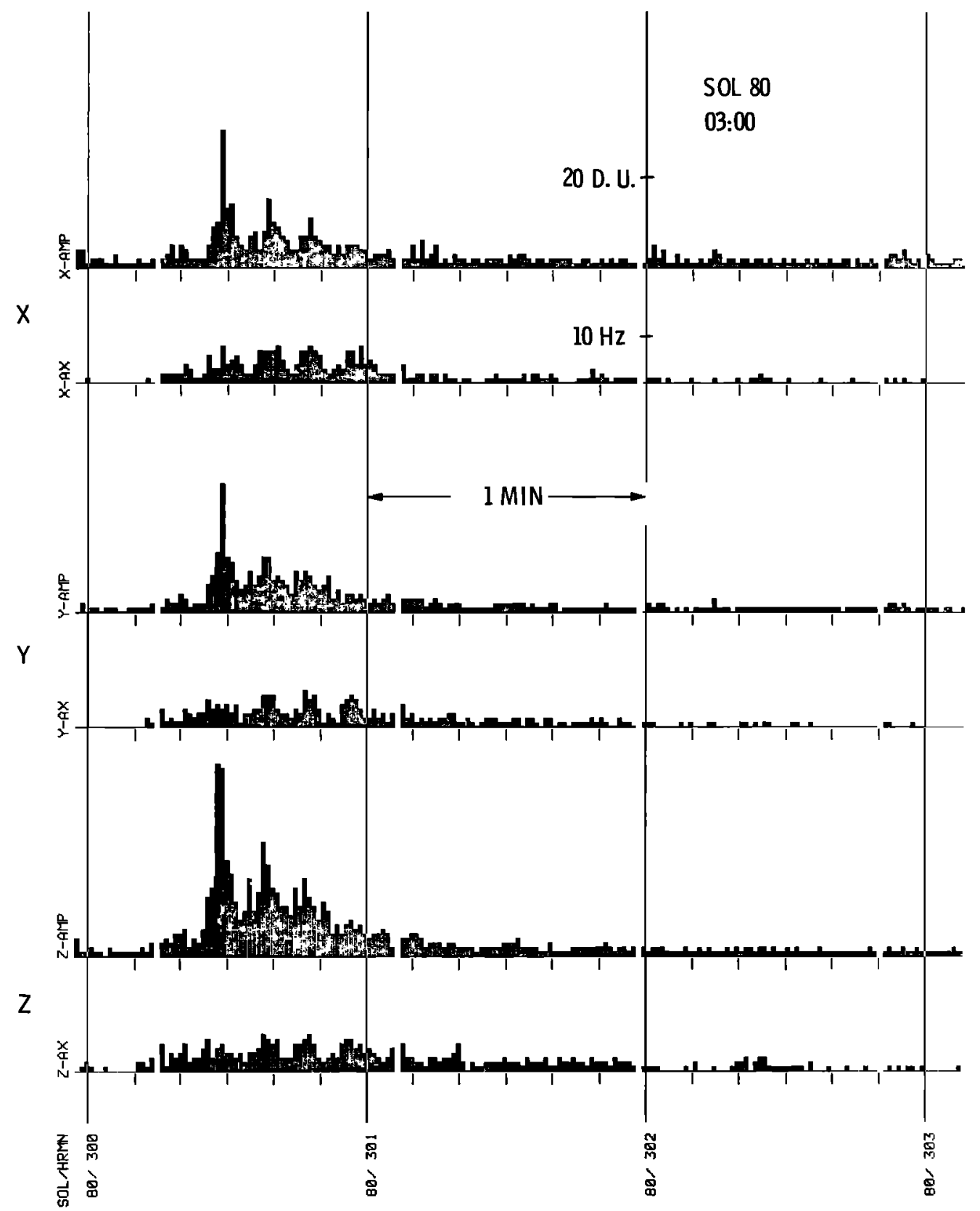

Fig. 21. Sol 800300 LLT event. This event is the most likely candidate for a Marsquake recorded to date. Because wind data were not recorded at the time of this event, it is possible that this event could also have been generated by the wind. 
TABLE 3. Times, Average Frequencies, and Wind Speeds for Eight Seismic Signals

\begin{tabular}{cccc}
\hline Sol & $\begin{array}{c}\text { Local Lander } \\
\text { Time, hours }\end{array}$ & $\begin{array}{c}\text { Average Signal } \\
\text { Frequency, Hz }\end{array}$ & $\begin{array}{c}\text { Maximum Wind } \\
\text { Speed, m/s }\end{array}$ \\
\hline 12 & 1650 & 3.48 & no data \\
23 & 1515 & 3.60 & 12 \\
42 & 1554 & 3.90 & 11 \\
49 & 1345 & 3.73 & 12 \\
49 & 1435 & 2.98 & 8 \\
49 & 1548 & 3.50 & 10 \\
53 & 1332 & 3.45 & 10 \\
80 & 0300 & 4.80 & no da1a \\
\hline
\end{tabular}

of the sol 80 event is therefore consistent with the amplitude for a Marsquake at a distance of $110 \mathrm{~km}$ in a crust having scattering and attenuation properties similar to those of the earth's crust.

There are at least two amplitude peaks (peaks 2 and 3) following the main peak at intervals of $10 \mathrm{~s}$ (peak 1 to peak 2) and $9 \mathrm{~s}$ (peak 2 to peak 3 ). One way to explain this pattern is by shear wave reflections in a surface layer. Many investigators have recognized such reflections in interpretation of seismograms and have used them in determination of crustal structure [e.g., Gutenberg. 1944]. If the two-way travel time for a shear wave traveling vertically in a surface layer is taken to equal $9 \mathrm{~s}$ and the layer shear wave velocity to equal $3.5 \mathrm{~km} / \mathrm{s}$, this interpretation gives a discontinuity at a depth of approximately $16 \mathrm{~km}$ beneath the region of landing site 2 . The peak amplitude ratios and assumed $Q$ of the layer can be used to calculate the reflection coefficient of the discontinuity. However, further speculation concerning the signal is of little value unless additional signals possessing similar characteristics are obtained.

The importance of positive identification of even a single event as being of internal (Marsquake) origin cannot be over-

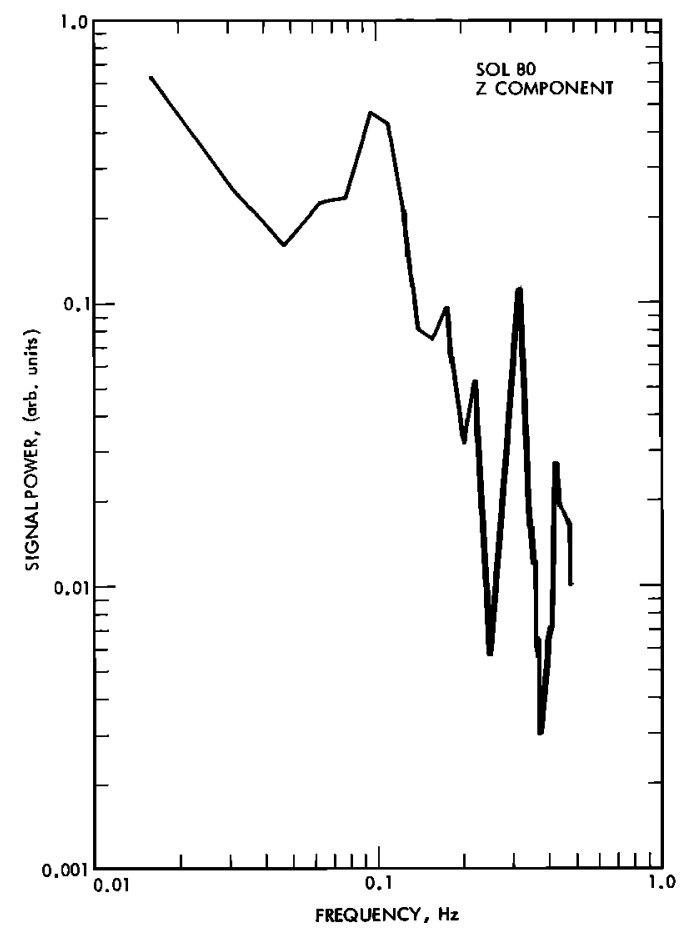

Fig. 22. Spectrum of the event mode amplitude envelope for the sol 80 event. The 10-s periodicity observed in the time domain is clearly visible in the spectrum.

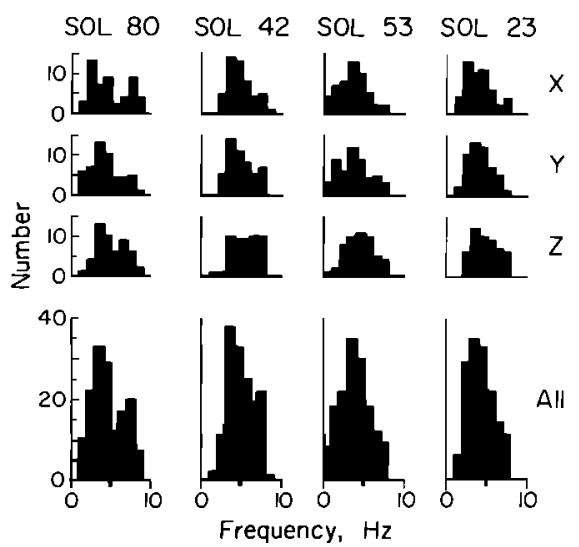

Fig. 23. Histogram of the sol 80 axis crossings compared with histograms from three other events. The sol 80 event is noticeably richer in high frequencies.

stressed. Aside from possible structural interpretations of the type described above, much can be learned from signal characteristics alone. For example, if the sol 80 event is a Marsquake, the similarities in signal characteristics between it and the terrestrial cases would indicate that the source parameters and transmission characteristics of Mars and earth are similar. This would be in marked contrast to the lunar case, in which signals are prolonged, with little coherence between the three components of ground motion. This is ascribed primarily to the very high $Q$ of the outer shell of the Moon and a sharp increase in velocity with depth in the superficial (regolith) zone. A moonquake with magnitude and range equal to those of the cases discussed above would have generated a seismic signal lasting several hours instead of $1 \mathrm{~min}$. If similarity between Martian and terrestrial signals can be established, we can say immediately that a lunarlike regolith is unlikely on Mars and that absorption of seismic wave energy is much higher in the region of Mars traversed by the observed signal. The high $Q$ in the case of the moon can be attributed to the absence of volatiles in the outer layers.

In summary, during the first 146 sols of operation of the Viking 2 seismometer, only one event has been detected that might be a local Marsquake. No events that can be interpreted as large teleseismic Marsquakes have been identified with certainty. These conclusions are based on visual inspection of the records. Only limited digital processing has been done to date. It should be pointed out that the seismometer has only been operating at highest gain and in the optimal modes (high data rate or event mode) during quiet periods, uncontaminated by lander activities or winds, for a cumulative total of about 30 days. This is too short a period of time to draw definitive conclusions about the seismicity of Mars. It should also be pointed out again that the seismicity of Mars is likely to be nonuniform both in time and space, as it is on the earth and the moon, and that the Viking 2 landing site is remote from the possibly most active regions of the planet inferred from photogeology and gravity information.

\section{Detection Capability, Seismicity, and METEOROID IMPACTS}

The detection of Marsquakes by the Viking seismometer system depends on the size and distance of the event, the magnification (gain level) of the instrument, and the background noise level. The noise level is discussed extensively elsewhere in this report. Furthermore, the amplitude of the 

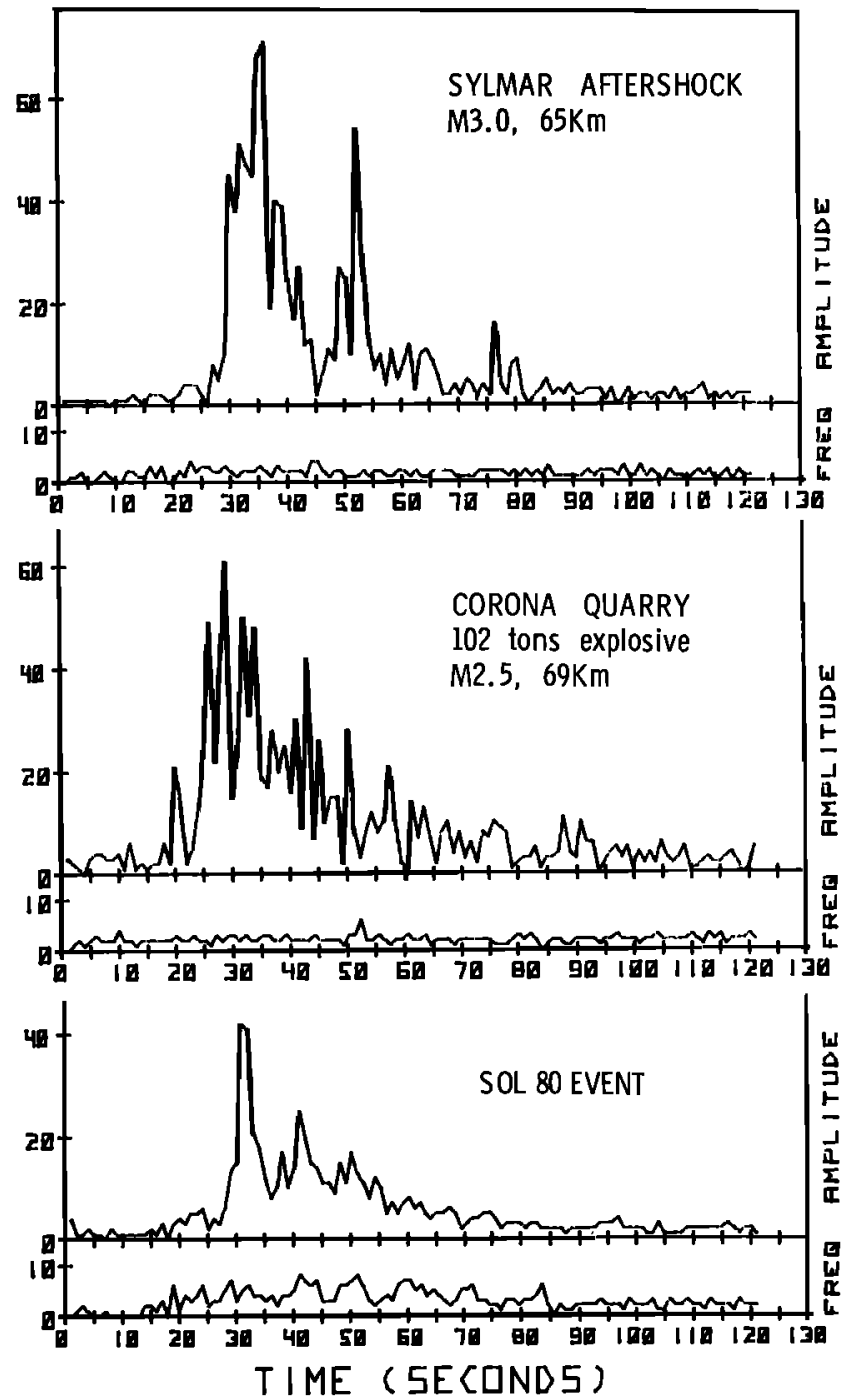

EVENT MODE COMPRESSED DATA

Fig. 24. The sol 80 event compared with similar events recorded in southern California by a Viking seismometer located at the California Institute of Technology. All events were recorded in the event mode.

ground motion produced at the Viking site from a given Marsquake depends on the velocity structure and the attenuation characteristics of the Martian interior. The internal structure models calculated on the basis of available constraints predict the presence of a core shadow zone starting at about $90^{\circ}-120^{\circ}$. The seismic ray paths for one such Mars model are shown in Figure 1.

The event detection capability of the Viking seismic instrument has been determined by combining the actual test data obtained in the earth and the ray theoretical amplitudes of seismic body waves calculated for a theoretical model of Mars. An average mantle $Q$ value of 2000 was assumed. The detection threshold as a function of distance is shown in Figure 26. This curve is based on the condition that an event can be identified if it produces a ground motion equivalent to 25 du or greater in the output seismic data. This is indeed a conservative case, since during wind-free periods the background noise rarely exceeds $3 \mathrm{du}$. Figure 26 shows that the Viking seismometer should detect Marsquakes of magnitude 3 at a distance of $200 \mathrm{~km}$, magnitude 5 at $1000 \mathrm{~km}$, and magni- tude 6 over half the surface of the planet $\left(\Delta=90^{\circ}\right)$. It is likely that an event of magnitude 6.5 or greater occurring anywhere on the surface of the planet can be detected by the Viking seismic instrument when the winds are relatively low.

To evaluate the level of seismic activity on Mars, it is necessary to analyze the available data, taking into account the operation modes of the instrument. The Marsquake detection capability of the Viking seismograph system has not been uniform throughout this period of observation because of the variability of wind interference, other lander activities, and limited data allocations. The detection capability is highest when the instrument is operated either in the high data rate or event mode, the level of wind-generated noise is less than 1-2 $\mathrm{du}$, and there are no other interfering lander activities. Under these conditions, seismic signals of amplitudes greater than 25 du should easily be identified. These conditions were met for a total of 640 hours during the first 146 sols of operation, or about $18 \%$ of the total time of operation.

On the basis of these data the comparative seismicity of Mars relative to that of the earth and moon can be estimated. On the earth, there are about 45 earthquakes per year of magnitude 6.5 or greater. Most earthquakes are at shallow depths. If the average number of Marsquakes per unit area of the Martian surface were the same as that on the earth, with the detection capability of the Viking seismometer one would expect to detect 13 Marsquakes of magnitude 6.5 or greater per year, or approximately one per month. No such events have yet been recognized during the 640 hours of the highsensitivity low-noise recording period of the Viking mission. From this limited sample it appears that Mars is seismically less active than the earth.

A more quantitative estimate of comparative seismicity of Mars relative to that of the earth and moon can be made by taking into account smaller events. If we assume that Marsquakes above a certain magnitude, like earthquakes, can be

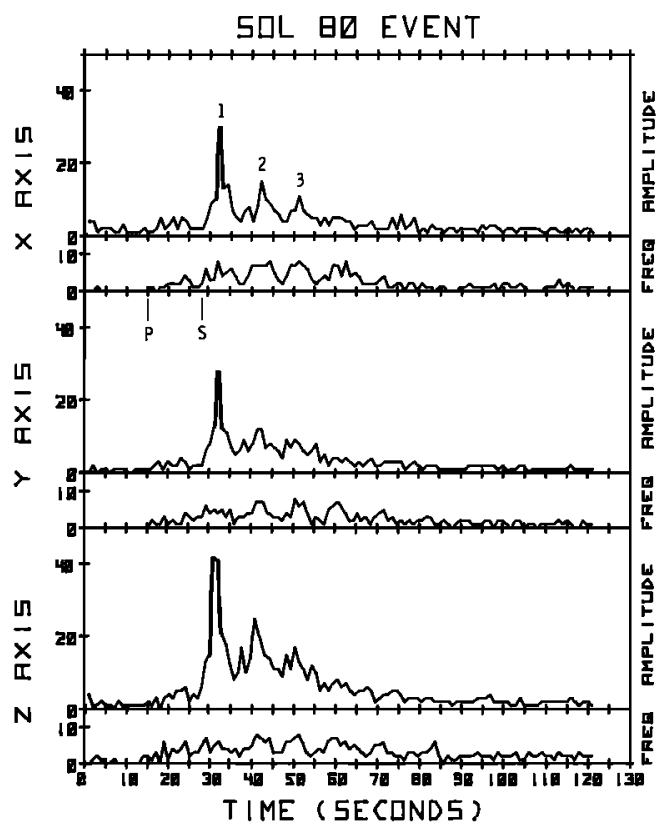

Fig. 25. The sol 80 event as recorded on all three axes. The $P$ and $S$ refer to the possible arrivals of compressional and shear waves. The peaks labeled 2 and 3 are possible reflections from the base of the crust. 


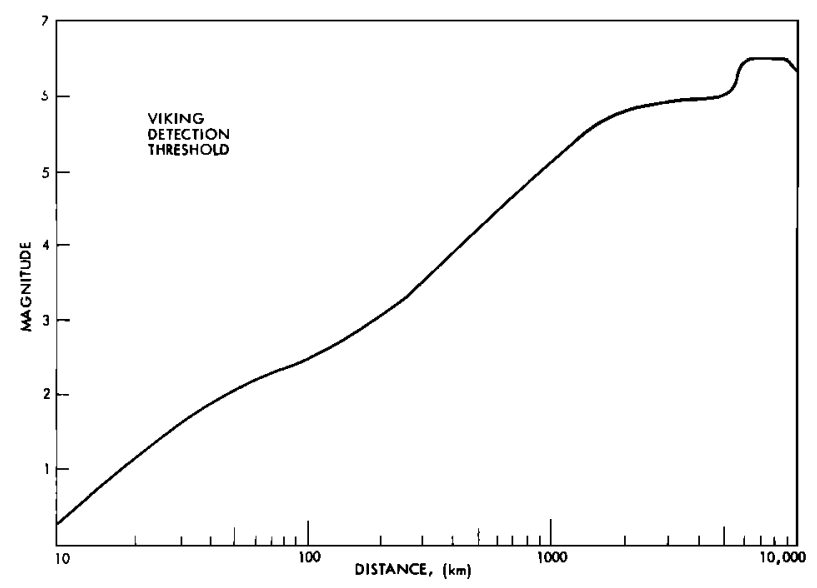

Fig. 26. Hypothetical Marsquake detection threshold values based on an average mantle $Q$ of 2000 and a detection threshold amplitude of $25 \mathrm{du}$. It is likely that a Marsquake of magnitude 6.5 or larger occurring anywhere on Mars could be detected.

represented by a random Poisson process, the probability of observing $n$ events over a period interval $T$ is

$$
P[n, T]=\left[(\alpha T)^{n} / n !\right] e^{-\alpha T}
$$

and the probability of observing no events is

$$
P[0, T]=e^{-\alpha T}
$$

where $\alpha$ is the mean rate of occurrence of events. Substituting the above mentioned values into this equation, we obtain

$$
P[0,640]=0.39
$$

Therefore although it is more probable that Mars is less active than the earth, there still is a $39 \%$ probability that Mars can be as active as the earth if the landing site happens to be an aseismic region of Mars. If we consider events of magnitude 4 or greater and if we assume that Marsquakes are uniformly distributed in space (thus assuming the nonexistence of aseismic regions), the number of expected Marsquakes will be increased to about 40 per year at earth rate. Therefore the probability of not detecting any event during 640 hours of observation under ideal conditions becomes

$$
P[0,640]=0.05
$$

Thus with the data collected so far and with the assumptions stated, the seismicity of Mars per unit surface area is less than that of the earth with a probability of $0: 95$. The additional data that will be collected during the next 2 years clearly will place more definite limits on Martian seismicity. It is also probable, as we discussed earlier, that the seismicity of Mars is not uniformly distributed in space and that the Viking 2 site may be in a relatively aseismic part of the planet.

A comparison of Martian seismicity with lunar seismicity can be made by using the data obtained from the seismometers deployed on the lunar surface during the Apollo mission [Latham et al., 1973]. The four seismic stations that are currently in operation are detecting moonquakes and meteoroid impact signals at a rate of about 2000 per year. The high rate of detection, however, is not due to the high seismicity of the moon but is due primarily to the high sensitivity of the instruments, the low background noise level, and the low attenuation characteristics of the lunar interior.

Correcting for the difference in instrument response, a Viking seismometer deployed at the Apollo 14 landing site would have detected three high-frequency transient events (possible shallow moonquakes at large distances [Nakamura et al., 1974]) and one meteoroid impact during the 6 years of operation. Multiplying by a factor of 4 to account for the difference in the surface areas of the moon and Mars, we would thus expect to have two Marsquakes of detectable sizes annually if Martian seismicity were the same as lunar seismicity and if the $Q$ of the Martian interior were as high as that of the lunar mantle, which is about 4000 [Dainty et al., 1976; Nakamura et al., 1976]. If we use statistics similar to those above, the chance that no Marsquakes are observed to date when an average 22 Marsquakes are expected per year (i.e., activity an order of magnitude higher than that on the moon) would be $20 \%$. Thus for a Mars of low attenuation comparable to the Moon we can say with $80 \%$ confidence that the seismicity of Mars cannot be greater than an order of magnitude above the lunar seismicity.

With the presence of volatiles the $Q$ of the Martian interior most likely is lower than that of the moon. If we assume a $Q$ of 500 , the detection of moonquake-sized events will be limited to within $500 \mathrm{~km}$ of a station. Then if we assume uniform areal distribution of Marsquakes, the expected number of detectable Marsquakes would be reduced to 1 in about 50 years if the Martian seismicity were the same as the lunar seismicity.

In summary, the Viking seismic observations to date are not sufficient to determine whether Mars is more active than the moon.

We next examine the question of the expected rate of detection of meteoroid impacts on Mars. Approximately 300 meteoroid impacts are detected on the lunar surface each year by the instruments of the Apollo Seismic Network [Duennebier et al., 1976]. These fall in the mass range from about $0.5 \mathrm{~kg}$ to 1 metric ton. Most estimates of meteoroid flux in the vicinity of Mars are equal to or larger than those in lunar space, so we might reasonably ask, Where are the impact signals on Mars?

Three factors reduce the likelihood of impact detection on Mars in relation to the moon. First, according to the theoretical results of Gault and Baldwin [1970] the Martian atmosphere is surprisingly effective as a shield against meteoroid impacts. Their results indicate that the number of meteoroids of $10-\mathrm{kg}$ mass reaching the surface would be only $10 \%$ of the number incident on the atmosphere, and the velocity of those that reach the surface would be reduced to several hundred meters per second. In fact, the impact velocity of objects as large as 1 metric ton will be significantly reduced. The predicted shielding from objects in the small to intermediate mass range is consistent with the angular appearance of the surface features of Mars compared with the much more subdued lunar morphology. A second factor bearing on relative rates of meteoroid detection is instrument sensitivity. The peak sensitivity of the Viking seismometer is about 70 times lower than that of the short-period Apollo seismometers. If the Apollo instruments had been operating at the sensitivity of the Viking seismometers, only one impact would have been recorded during the entire period of operation of the Apollo network (7 yr).

Finally, the influence of seismic wave absorption must be considered. The $Q$ of the outer shell of the moon has been estimated to be about 6000 [Latham et al., 1973]. Typical terrestrial values for shallow depths range from 200 to 300 . This difference is ascribed primarily to the quantity of water contained in the medium: reduced water content resulting in higher $Q$. On this basis we might expect $Q$ values in the outer shell of Mars to be much lower than those found in the moon but possibly higher than terrestrial values. 
If we assume a Mars $Q$ of 500 and a moon $Q$ of 6000 , the attenuation of a seismic wave at a range of $2000 \mathrm{~km}$ will be 40 times greater for Mars than for the moon.

If we take these three factors (atmospheric shielding, instrument sensitivity, and attenuation) together, the likelihood of detecting a meteoroid impact on Mars with the Viking seismometers during the planned lifetime of the experiment is extremely small.

\section{IMPLICATIONS FOR FUTURE MisSIONS}

The Viking experiment, even though it was highly curtailed by mission priorities and by the inability to uncage the first seismometer, has provided and will continue to provide valuable information for the planning of any future missions to Mars. First, it established that the seismic background noise on Mars due to winds and atmospheric pressure fluctuations is very low. The Viking seismometer, mounted on a relatively compliant spacecraft with a large surface area exposed to winds, could still operate at maximum sensitivity at least half of the time during the nominal mission, a situation that indicated that seismometers with much greater sensitivities can be operated on the planet. Emplaced by penetrators or deployed as small packages, seismometers more sensitive than the Viking instrument by a factor of at least $10^{3}$ can operate on the planet without being affected by typical Martian winds.

Although the data on Martian seismicity are still preliminary, the indications are that Mars is probably less active than the earth. Greater sensitivity is a must for any future seismic instruments on the planet.

Another important consideration is the deployment of a network of instruments. With a well-placed network of five very sensitive A pollo seismographs it was possible to study the moon and determine its structure, even though most moonquakes are very small. A similar approach of deployment of a network of highly sensitive instruments is needed for Mars exploration. Since Mars is a larger planet than the moon and shows much greater geologic and tectonic diversity, both global and regional seismic networks are needed to understand Martian structure and tectonics. It is important that future missions deploy such networks.

Acknowledgments. The Viking seismometer was designed and tested by F. L. Lehner and W. Miller at the California Institute of Technology. Flight hardware was designed and fabricated by $J$. Lewko, D. Gibson, M. Van Dyke, T. Gaffield, and D. LaFeniere of Bendix Aerospace. The large-scale integration circuitry was fabricated by American Microsystems, Inc. Wyatt Underwood has been in charge of downlink operations. George Sutton and Ken Anderson were involved in various aspects of the experiment. We thank $W$. Kaula, R. Phillips, B. Bills, A. Ferrari, and E. Okal for useful discussions and for some of the calculations given in this paper. The cooperation of the Meteorology Team headed by S. Hess is gratefully acknowledged. This work was supported by NASA contract NAS19703. Contribution 2910 of the Division of Geological and Planetary Sciences, California Institute of Technology, Pasadena, Californa 91225 .

\section{REFERENCES}

Anderson, D. L., Implications of the inhomogeneous planetary accretion hypothesis, Comments Earth Sci. Geophys., 2, 93-98, 1971.

Anderson, D. L., Internal constitution of Mars, J. Geophys. Res., 77. 789-795, 1972.

Anderson, D. L., The moon as a high temperature condensate, Moon, $8,33-57,1973$.

Anderson, D. L., and R. L. Kovach, The composition of the terrestrial planets, Earth Planet. Sci. Lett., 3, 19-24, 1967.

Anderson, D. L., R. L. Kovach, G. Latham, F. Press, M. N. Toksöz, and G. Sutton, Seismic investigations: The Viking Mars lander, Icarus, 16, 205-216, 1972.
Anderson, D. L., F. K. Duennebier, G. V. Latham, M. N. Toksöz, R. L. Kovach, T. C. D. K night, A. R. Lazarewicz, W. F. Miller, Y. Nakamura, and G. Sutton, The Viking seismic experiment, Science, 194, 1318-1321, 1976.

Bills, B. G., and A. J. Ferrari, Mars topography harmonics and geophysical implications, J. Geophys. Res., 82, in press, 1977a.

Bills, B. G., and A. J. Ferrari, A lunar density model consistent with topographic, gravitational, librational, and seismic data, $J$. Geophys. Res., 82, 1306-1314, $1977 b$.

Binder, A. B., Internal structure of Mars, J. Geophys. Res., 74, 3110-3117, 1969.

Binder, A. B., and D. R. Davis, Internal structure of Mars, Phys. Earth Planet. Interiors, 7. 477-485, 1973.

Blasius, K. R., and J. A. Cutts, Shield volcanism and lithospheric structure beneath the Tharsis plateau, Mars, Proc. Lunar Sci. Conf. 7th, 3, 3561-3574, 1976.

Dainty, A. M., M. N. Toksöz, and S. Stein, Seismic investigation of the lunar interior, Proc. Lunar Sci. Conf. 7th, 3057-3075, 1976.

Duennebier, F., Y. Nakamura, G. Latham, and H. Dorman, Meteoroid storms detected on the moon, Science, 192, 1000-1002, 1976.

Gault, D., and B. Baldwin, Impact cratering on Mars-Some effects of the atmosphere (abstract), Eos Trans. AGU, 5I, 343, 1970.

Gutenberg, B., Reflected and minor phases in records of near-by earthquakes in southern California, Bull. Seismol. Soc. Amer., 34, 137-160, 1944.

Hess, S. L., R. M. Henry, C. B. Leovy, J. A. Ryan, and J. E. Tillman, Meteorological results from the surface of Mars: Viking 1 and 2, J. Geophys. Res., 82, this issue, 1977.

Johnston, D. H., and M. N. Toksöz, Internal structure and properties of Mars, Icarus, in press, 1977.

Johnston, D. H., R. T. McGetchin, and M. N. Toksöz, Thermal state and internal structure of Mars, J. Geophys. Res., 79, 3959-3971, 1974.

Kovach, R. L., and D. L. Anderson, The interiors of the terrestrial planets, J. Geophys. Res., 70, 2873-2882, 1965.

Latham, G. V., M. Ewing, F. Press, J. Dorman, Y. Nakamura, M. N. Toksöz, D. Lammlein, F. Duennebier, and A. M. Danty, Passive seismic experiment, Apollo 17 Preliminary Science Report, NASA Spec. Publ., 330, 1-9, 1973.

Lee, B. G., Mission operations strategy for Viking, Science, 194. $59-62,1976$.

Nakamura, Y., J. Dorman, F. Duennebier, M. Ewing, D. Lammlein, and G. V. Latham, High frequency lunar teleseismic events, Proc. Lunar Sci. Conf. Sth, 3, 2883-2890, 1974.

Nakamura, Y., F. Duennebier, G. V. Latham, and J. Dorman, Structure of the lunar mantle, J. Geophys. Res., 81, 4818-4824, 1976.

Okal, E., and D. L. Anderson, Seismic models for Mars, submitted to Icarus, 1977

Owen, T., and K. Biemann, Composition of the atmosphere at the surface of Mars: Detection of argon 36 and prelıminary analysis, Science, 193, 801-803, 1976.

Phillips, R. J., and R. S. Saunders, The isostatic state of Martian topography. J Geophys. Res., 80, 2893-2897, 1975.

Phillips, R. J., and M. Tiernan, Martian stress distributions: Arguments against the static support of Tharsis, submıtted to J. Geophys. Res, 1977.

Phillips, R. J., R. S. Saunders, and J. E. Conel, Mars: Crustal structure inferred from Bouguer gravity anomalies, J. Geophys. Res., 78, 4815-4820, 1973.

Reasenberg, R., The moment of inertia and isostasy of Mars, $J$. Genphys. Res.. 82, 369-375, 1977.

Ringwood, A. E., and S. P. Clark, Internal constitution of Mars, Nature, 234, 89-92, 1971.

Toksöz, M. N., and D. H. Johnston, The evolution of the moon and the terrestrial planets, in The Proceedings of the Soviet-American Conference on the Cosmochemistry of the Moon and Planets, in press, 1977.

Toksöz, M. N., A. M. Dainty, S. C. Solomon, and K. R. Anderson, Structure of the moon, Rev. Geophys. Space Phys., 12, 539-567, 1974.

Urey, H. C., The Planets, Their Origin and Development, Yale University Press, New Haven, Conn., 1952.

(Received March 30, 1977; revised May 9, 1977; accepted May 9, 1977.) 